\title{
Testing capability indices for manufacturing processes with asymmetric tolerance limits and measurement errors
}

\author{
D. $\mathrm{Grau}^{\star}$ \\ Laboratory of Applied Mathematics, CNRS UMR 5142, IUT de Bayonne, Université de Pau et des Pays de l'Adour, \\ 17 Place Paul Bert, 64100 Bayonne, France
}

Received: 24 January 2011 / Accepted: 3 March 2011

\begin{abstract}
Most research works related to process capability indices assume no gauge measurement errors. However, such an assumption inadequately reflects real situations even when advanced measuring instruments are employed. If we do not take into account these errors, conclusions drawn from process capability are therefore unreliable. In this paper we study the sampling distribution of capability indices $C_{p}^{\prime \prime}(u, v)$ in the presence of measurements errors, and when small subsamples data are collected from past "in-control". We show that using a critical value without taking into account these errors, severely underestimates the $\alpha$-risk which causes a less accurate testing capacity. To improve the results we suggest the use of an adjusted critical value, and we give a Maple program to get it. An example in a nougat manufactory is presented to illustrate this approach.
\end{abstract}

Keywords: Process capability indices; gauge measurement error; asymmetric tolerances; critical value; power test

\section{Introduction}

Process capability indices, which provide numerical measures on whether a process meets the capability requirement preset in the factory, have been widely applied to the industry for evaluating manufacturing performance. Recent research in numerous articles and several books such as Kotz and Johnson [1], Bothe [2], Kotz and Lovelace [3], or Pearn and Kotz [4] have focussed on the study of these capability indices. The original process capability index is $C_{p}$, which is designed to provide a measure of potential ability to meet requirements. The major weakness of this index lies in the fact that it does not consider the mean of the process. Kane [5] considered the $C_{p k}$ index in order to show the influence of a shift of the process mean on the ability of the process to produce products within the tolerance values. The $C_{p m}$ index suggested by Chan et al. [6] involves the variation of production items with respect to the target value and the tolerance limits that are preset in the factory. Combining the advantages of these previous indices, Pearn et al. [7] introduced the $C_{p m k}$ index. For a process with lower and upper tolerance limits $L S L$ and $U S L$, and a target $T$ set to the midpoint $m=(L S L+U S L) / 2$ of the tolerance interval, Vännman [8] constructed a unified superstructure for the

^ Correspondence: daniel.grau@univ-pau.fr four previous basic indices which can be defined as

$$
C_{p}(u, v)=\frac{d-u|\mu-m|}{3 \sqrt{\sigma^{2}+v(\mu-T)^{2}}},
$$

where $d=(U S L-L S L) / 2$ is the half-length of the tolerance interval, $\mu$ is the mean, $\sigma$ is the standard deviation, and $u$ and $v$ are two non-negative parameters. It is easy to verify that $C_{p}=C_{p}(0,0), C_{p k}=C_{p}(1,0), C_{p m}=C_{p}(0,1)$, and $C_{p m k}=C_{p}(1,1)$.

A process is said to have symmetric tolerances if the target value $T$ is set to the midpoint $m$ of the tolerance interval, that is to say $T=m$. Although cases with symmetric tolerances are common in practical situations, cases with asymmetric tolerances often occur in manufacturing industry. In general asymmetric tolerances reflect that deviations from the target are less tolerable in one direction than in the other. Nevertheless, asymmetric tolerances can also arise in those situations where the tolerances are symmetric to begin with, but the process distribution following a non-normal distribution, the data are transformed to achieve approximate normality. If indices $C_{p}(u, v)$ are well adapted to the case of symmetrical tolerances, they have some undesirable properties when the tolerances are asymmetrical, $T \neq m$ (see Boyles [9]). To overcome the problems with asymmetric tolerances, and to generalize the family $C_{p}(u, v)$ to the case $T \neq m$, Chen and Pearn [10] suggested using the family

$$
C_{p}^{\prime \prime}(u, v)=\frac{d^{*}-u A^{*}}{3 \sqrt{\sigma^{2}+v A^{2}}},
$$


where $A=\max \left\{d(\mu-T) / D_{u}, d(T-\mu) / D_{l}\right\}, A^{*}=$ $\max \left\{d^{*}(\mu-T) / D_{u}, d^{*}(T-\mu) / D_{l}\right\}, \quad D_{u}=U S L-$ $T, \quad D_{l}=T-L S L$, and $d^{*}=\min \left\{D_{u}, D_{l}\right\}$. Grau [11] suggests choosing parameters $u$ and $v$ according to the importance the user attaches to the process yield, or to the process centring, which are the most important criteria to measure the process performance.

In practice, process capability indices are unknown and are estimated from data obtained from samples of manufactured items. Therefore, a certain amount of uncertainty, due to the sampling error, is necessarily present in the evaluation of the process performance. A further source of uncertainty is given by the measurements errors even with highly advanced measuring instruments. Montgomery and Runger $[12,13]$ pointed out that quality of the collected data on the process characteristics mostly relies on the gauge accuracy. Levinson [14] and Mittag [15] were the first to quantify the percentage error on process capability indices evaluation in the presence of measurement errors. Bordignon and Scagliarini [16-18] study the statistical properties of the estimators of $C_{p}, C_{p k}$, and $C_{p m}$ in the case of measurement errors. For the $C_{p}, C_{p k}$, $C_{P U}, C_{P L}, C_{p m}$ and $C_{p m k}$ indices, Pearn and Liao [19-21], Pearn et al. [22], Hsu et al. [23] present some statistical properties and establish reliable lower confidence bounds and reliable critical values to estimate and test the process capability with gauge measurements errors.

The purpose of this paper is to construct a critical value to test whether a process is capable when gauge measurement errors are present. This article is organized as follows. In Section 2, for processes with symmetrical or asymmetrical tolerance limits, we quickly report the main results concerning the effects of gauge measurement errors on theoretical capability indices. In Section 3 we give the sampling distribution of capability indices in the presence of measurements errors, and when small subsamples data are collected from past "in-control". Sections 4 and 5 give critical values and adjusted critical values to test process capability when gauge measurement error is present. Finally, a real example is presented in the last section.

\section{The index $C_{p}^{\prime \prime}(u, v)$ and the gauge measurement errors}

Suppose that the gauge measurement errors can be described as a random variable $M \square N\left(0, \sigma_{M}^{2}\right)$. Montgomery and Runger [12] expressed the gauge capability as

$$
\lambda=\frac{6 \sigma_{M}}{U S L-L S L} .
$$

In this paper, the gauge capability provided by the gauge manufacturing factory is assumed to be known. Suppose that $X \square N\left(\mu, \sigma^{2}\right)$ represents the relevant quality characteristic of the manufacturing process. In practice the observed variable $G$ (with gauge measurement errors) is measured rather than the true variable $X$. It is further assumed that $X$ and $M$ are additively linked according to
$G=X+M$ and that $X$ and $M$ are stochastically independent. Then we have $G \square N\left(\mu, \sigma_{G}^{2}=\sigma^{2}+\sigma_{M}^{2}\right)$ and the empirical process capability index $C_{p}^{\prime \prime} G(u, v)$ is obtained by formula (1) after substituting $\sigma_{G}$ for $\sigma$.

$$
C_{p}^{\prime \prime G}(u, v)=\frac{d^{*}-u A^{*}}{3 \sqrt{\sigma_{G}^{2}+v A^{2}}} .
$$

Obviously if $\sigma_{M}=0$, then the empirical process capability $C_{p}^{\prime \prime G}(u, v)$ reduces to the basic index $C_{p}^{\prime \prime}(u, v)$. Since the variation of the observed data is larger than the variation of the original data, the denominator of the index $C_{p}^{\prime \prime G}(u, v)$ becomes larger and we would understate the true capability of the process if the empirical data $G$ are used.

Let $\delta=(T-m) / d$ be the quantity that allows locating the position of $T$ in the tolerance interval. We have $d / D_{u}=(1-\delta)^{-1}, d / D_{l}=(1+\delta)^{-1}$, and $d^{*} / d=1-|\delta|$. Assume that $C_{p}=C_{p}(0,0)$ and $C_{p}^{\prime \prime}=C_{p}^{\prime \prime}(0,0)$. Since $\lambda C_{p}^{\prime \prime} /(1-|\delta|)=\lambda C_{p}=\sigma_{M} / \sigma$, the relationship between the true process capability, $C_{p}^{\prime \prime}(u, v)$, and the empirical process capability, $C_{p}^{\prime \prime G}(u, v)$, can be expressed as

$$
C_{p}^{\prime \prime G}(u, v)=\frac{\sqrt{1+v \xi^{* 2}}}{\sqrt{1+\lambda^{2} C_{p}^{\prime \prime 2} /(1-|\delta|)^{2}+v \xi^{* 2}}} C_{p}^{\prime \prime}(u, v),(3)
$$

where $\xi^{*}=\max \left((1-\delta)^{-1} \xi,-(1+\delta)^{-1} \xi\right)$ and $\xi=$ $(\mu-T) / \sigma$. It is clear that the ratio $C_{p}^{\prime \prime G}(u, v) / C_{p}^{\prime \prime}(u, v)$ is decreasing function of $\lambda$ and $C_{p}^{\prime \prime}$. Therefore the measurements errors underestimate the true theoretical capability of the process, especially when the potential capability $C_{p}^{\prime \prime}$ is large. However, the theoretical capability is unknown and is estimated from sample data. Thus, in the following sections we deal with the effects of measurement errors on the performance of estimated capability indices.

\section{Sampling distribution of $\hat{C}_{p}^{\prime \prime G}(u, v)$}

A common practice of the process capability estimation in the manufacturing industry is to first implement a routine-based data collection program for monitoring the process stability. In order to analyze the past "in control" data, $r$ subgroups with variable sample sizes $n_{i}$, $\left(X_{i 1}, X_{i 2}, \ldots, X_{i n_{i}}\right)$, are selected randomly from a stable process following a normal distribution $N\left(\mu, \sigma^{2}\right)$. Let $\bar{X}_{i}=\sum_{j=1}^{n_{i}} X_{i j} / n_{i}$ and $S_{i}=\left[n_{i}^{-1} \sum_{j=1}^{n_{i}}\left(X_{i j}-\bar{X}_{i}\right)^{2}\right]^{1 / 2}$ be the $i$ th sample mean and the sample standard deviation, respectively, and $N=\sum_{i=1}^{r} n_{i}$ the total number of observations. We consider the following natural estimator of $C_{p}^{\prime \prime}(u, v)$ defined as

$$
\hat{C}_{p}^{\prime \prime}(u, v)=\frac{d^{*}-u \hat{A}^{*}}{3 \sqrt{S^{2}+v \hat{A}^{2}}}
$$

where $\hat{A}=\max \left\{(1-\delta)^{-1}(\overline{\bar{X}}-T),(1+\delta)^{-1}(T-\overline{\bar{X}})\right\}$, $\hat{A}^{*}=\left(d^{*} / d\right) \hat{A}=(1-|\delta|) \hat{A}, \overline{\bar{X}}=\sum_{i=1}^{r} n_{i} \bar{X}_{i} / N$, and 


$$
\xi^{*}=\frac{-C_{p}^{\prime \prime} u(1-|\delta|) / 3+\sqrt{\left(C_{p}^{\prime \prime}\right)^{2}(u(1-|\delta|) / 3)^{2}+\left(\left(C_{p}^{\prime \prime}\right)^{2}-c^{2}\right)\left(v c^{2}-(u(1-|\delta|) / 3)^{2}\right)}}{v c^{2}-(u(1-|\delta|) / 3)^{2}} .
$$

$S^{2}=\sum_{i=1}^{r} n_{i} S_{i}^{2} / N$. We define $K=N S^{2} / \sigma^{2}, Z=N^{1 / 2}$ $(\overline{\bar{X}}-T) / \sigma$, and $Y=\left[\max \left\{(1-\delta)^{-1} Z,-(1+\delta)^{-1} Z\right\}\right]^{2}$. Then the estimator $\hat{C}_{p}^{\prime \prime}(u, v)$ can be rewritten as

$$
\hat{C}_{p}^{\prime \prime}(u, v)=\frac{3 \sqrt{N} C_{p}^{\prime \prime}-(1-|\delta|) u \sqrt{Y}}{3 \sqrt{K+v Y}} .
$$

Under the assumption of normality, $K$ is distributed as $\chi_{N-r}^{2}$, an ordinary central Chi-square distribution with $N-r$ degree of freedom, $Z$ is distributed as $N(\sqrt{N} \xi, 1)$ and $Y$ is distributed as $\chi_{1}^{2}\left(N \xi^{2},(1-\delta)^{-1},(1+\delta)^{-1}\right)$, an unbalanced non-central chi-square distribution with one degree of freedom and noncentrality parameter $N \xi^{2}$ (Grau [24]). Applying the same technique used in Pearn et al. [25] to obtain the cumulative distribution of $\hat{C}_{p m k}^{\prime \prime}=$ $\hat{C}_{p}^{\prime \prime}(1,1)$, for $(u, v) \neq(0,0)$ we have

$$
F_{\hat{C}_{p}^{\prime \prime}(u, v)}(x)=1-\int_{0}^{K(x)} H(x, t) d t, \quad \text { for } \quad x>0
$$

where $K(x)=b \sqrt{N} /((1-|\delta|) u+3 x \sqrt{v}), b=3 C_{p}^{\prime \prime}$,

$H(x, t)=F_{K}\left(\left(\frac{b \sqrt{N}-(1-|\delta|) u t}{3 x}\right)^{2}-v t^{2}\right) f_{Y}(t), \quad$ with $F_{K}(x)$ the cumulative distribution of $K, f_{Y}(t)=$ $(1-\delta) \phi((1-\delta) t-\sqrt{N} \xi)+(1+\delta) \phi((1+\delta) t+\sqrt{N} \xi)$, and $\phi(x)$ the probability density of the standard normal distribution $N(0,1)$.

When there are gauge measurement errors, variable $G$ is measured rather than the true variable $X$. $r$ subgroups $\left(G_{i 1}, G_{i 2}, \ldots, G_{i n_{i}}\right)$ are selected randomly from a stable process following a normal distribution $N\left(\mu, \sigma_{G}^{2}\right)$. We denote $\bar{G}_{i}=\sum_{j=1}^{n_{i}} G_{i j} / n_{i}, S_{G_{i}}=\left[n_{i}^{-1} \sum_{j=1}^{n_{i}}\left(G_{i j}-\bar{G}_{i}\right)^{2}\right]^{1 / 2}$, $\xi_{G}=(\mu-T) / \sigma_{G}, \xi_{G}^{*}=\max \left((1-\delta)^{-1} \xi_{G},-(1+\delta)^{-1} \xi_{G}\right)$, and consider the following natural estimator of $C_{p}^{\prime \prime G}(u, v)$ defined as

$$
\hat{C}_{p}^{\prime \prime G}(u, v)=\frac{d^{*}-u \hat{A}_{G}^{*}}{3 \sqrt{S_{G}^{2}+v \hat{A}_{G}^{2}}}
$$

where $\hat{A}_{G}=\max \left\{(1-\delta)^{-1}(\overline{\bar{G}}-T),(1+\delta)^{-1}(T-\overline{\bar{G}})\right\}$, $\hat{A}_{G}^{*}=\left(d^{*} / d\right) \hat{A}_{G}=(1-|\delta|) \hat{A}_{G}, \overline{\bar{G}}=\sum_{i=1}^{r} n_{i} \bar{G}_{i} / N$, and $S_{G}^{2}=\sum_{i=1}^{r} n_{i} S_{G_{i}}^{2} / N$. Thus the cumulative distribution of $\hat{C}_{p}^{\prime \prime G}(u, v)$ is

$$
F_{\hat{C}_{p}^{\prime \prime G}(u, v)}(x)=1-\int_{0}^{K_{G}(x)} H_{G}(x, t) d t, \quad \text { for } \quad x>0,
$$

where $K_{G}(x)=b_{G} \sqrt{N} /((1-|\delta|) u+3 x \sqrt{v})$, $b_{G}=3 C_{p}^{\prime \prime G}$ with $C_{p}^{\prime \prime G}=C_{p}^{\prime \prime G}(0,0), H_{G}(x, y)=$ $F_{K}\left(\left(\frac{b_{G} \sqrt{N}-(1-|\delta|) u t}{3 x}\right)^{2}-v t^{2}\right) f_{G}(t), \quad$ and $\quad f_{G}(t)=$ $(1-\delta) \phi\left((1-\delta) t-\sqrt{N} \xi_{G}\right)+(1+\delta) \phi\left((1+\delta) t+\sqrt{N} \xi_{G}\right)$.

\section{Capability testing based on $\hat{C}_{p}^{\prime \prime G}(u, v)$}

To determine whether a given process meets the preset capability requirement, we could consider the following statistical hypotheses testing:

- $H_{0}: C_{p}^{\prime \prime}(u, v) \leqslant c$ Process is not capable;

- $H_{1}: C_{p}^{\prime \prime}(u, v)>c$ Process is capable;

where $c$ is the required process capability. If the calculated process capability $\hat{c}_{p}^{\prime \prime}(u, v)$ is greater than the critical value $c_{0}$, we reject the null hypothesis and conclude that the process is capable with error $\alpha$, which is the chance of incorrectly concluding an incapable process as capable. Given values of $c$ and $\alpha$, from (4) the critical value $c_{0}$ can be determined by solving the equation $\alpha=P\left(\hat{C}_{p}^{\prime \prime}(u, v)>\right.$ $\left.c_{0} \mid C_{p}^{\prime \prime}(u, v)=c\right)=\int_{0}^{K\left(c_{0}\right)} H\left(c_{0}, t\right) d t$, where from (A.6) in Appendix A, $b=3 C_{p}^{\prime \prime}=3 \sqrt{1+v \xi^{* 2}} c+u(1-|\delta|) \xi^{*}$. However, since the process parameters $\mu$ and $\sigma$ are unknown, parameters $\xi$ and therefore $\xi^{*}$ are unknown. For the particular cases $C_{p k}, C_{p m}$, and $C_{p m k}$, Pearn and Lin [26], Lin and Pearn [27], Hsu et al. [23], performed extensive calculations to obtain the maximum value of the critical value and showed that its maximum is obtained for the values of $\xi$ equal respectively to 1,0 , and 0.5 . Then they used those previous values in order to eliminate the necessity to estimate $\xi$. It is not possible to use the same method for any $(u, v)$ pair, thus in the previous equations, we replace $\xi$ and $\xi^{*}$ by the observation of the natural estimators $\hat{\xi}=(\bar{X}-T) / S$ and $\hat{\xi}^{*}=\max \left((1-\delta)^{-1} \hat{\xi},-(1+\delta)^{-1} \hat{\xi}\right)$. Note that we use the same symbols $\hat{\xi}$ and $\hat{\xi}^{*}$ for the estimators as well as the estimations.

In the presence of measurements errors, $C_{p}^{\prime \prime G}(u, v)$ is measured rather than the true variable $C_{p}^{\prime \prime}(u, v)$. Thus the $\alpha$-risk denoted by $\alpha_{G}$ is defined as $\alpha_{G}=$ $P\left(\hat{C}_{p}^{\prime \prime G}(u, v)>c_{0} \mid C_{p}^{\prime \prime}(u, v)=c\right)$. Since the process capability index is estimated by using $\hat{C}_{p}^{\prime \prime G}(u, v)$ instead of $\hat{C}_{p}^{\prime \prime}(u, v)$, the real capability of the process is underestimated. The probability of $\hat{C}_{p}^{\prime \prime G}(u, v)$ being greater than $c_{0}$ will be less important than by using $\hat{C}_{p}^{\prime \prime}(u, v)$. Thus $\alpha_{G}$, the $\alpha$-risk using $\hat{C}_{p}^{\prime \prime G}(u, v)$ to estimate $C_{p}^{\prime \prime}(u, v)$ is less than $\alpha$, using $\hat{C}_{p}^{\prime \prime}(u, v)$ when estimating $C_{p}^{\prime \prime}(u, v)$. To illustrate the performance of $\alpha_{G}$ versus $\lambda$, we have considered the particular case $u=0.5, v=1.2, \delta=0.3, c=1$ and $1.5, \alpha=0.05$, and have plotted curves $\alpha_{G}$ for various $C_{p}^{\prime \prime}$ values. The critical value $c_{0}$ is obtained from (4) where $\xi$ is defined from (A.2) and $\xi^{*}$ is defined from (A.15) as

\section{See equation above.}

Then from (5) we compute $\alpha_{G}=\int_{0}^{K_{G}\left(c_{0}\right)} H_{G}\left(c_{0}, t\right) d t$, where $C_{p}^{\prime \prime G}$ and $\xi_{G}$ are obtained from (A.10) and (A.12). 
Figure 1 plots $\alpha_{G}$ versus $\lambda \in[0,0.5]$ with $r=1, N=30$, $50,70,100,150$ for $C_{p}^{\prime \prime}=c(0.5)(c+1)$. Note that for $\lambda=0$, $\alpha_{G}=\alpha$. In Figure $1, \alpha_{G}$ decreases as $\lambda$ or $N$ increases, and the decreasing rate is more significant with large $c$.

We now consider the power of the test, the chance of correctly concluding a capable process as capable. It can be computed as $\pi\left(C_{p}^{\prime \prime}(u, v)\right)=$ $P\left(\hat{C}_{p}^{\prime \prime}(u, v)>c_{0} \mid C_{p}^{\prime \prime}(u, v)\right)=\int_{0}^{K\left(c_{0}\right)} H\left(c_{0}, t\right) d t$, with from (A.6), $b=3 C_{p}^{\prime \prime}=3 \sqrt{1+v \xi^{*^{2}}} C_{p}^{\prime \prime}(u, v)+u(1-|\delta|) \xi^{*}$.

In the presence of measurements errors, the power of the test denoted by $\pi_{G}$ is as follows $\pi_{G}\left(C_{p}^{\prime \prime}(u, v)\right)=$ $P\left(\hat{C}_{p}^{\prime \prime G}(u, v)>c_{0} \mid C_{p}^{\prime \prime}(u, v)\right)=\int_{0}^{K_{G}\left(c_{0}\right)} H_{G}\left(c_{0}, t\right) d t$. To illustrate the performance of $\pi_{G}$ versus $\lambda$, we consider the particular case $u=0.5, v=1.2, \delta=0.3$, and plot the curves $\pi_{G}$ for various $C_{p}^{\prime \prime}$ and $C_{p}^{\prime \prime}(u, v)$ values. In the previous equation, $C_{p}^{\prime \prime G}, \xi^{*}, \xi$ and $\xi_{G}$ are obtained from (A.10), (A.15), (A.2) and (A.12). Figure 2 plots $\pi_{G}$ versus $\lambda \in[0,0.5]$ with $r=1, N=50, \alpha=0.05, c=1.00$ and $1.50, C_{p}^{\prime \prime}(u, v)=c(0.20)(c+1)$, and $C_{p}^{\prime \prime}=C_{p}^{\prime \prime}(u, v)$, $C_{p}^{\prime \prime}(u, v)+0.33$ and $C_{p}^{\prime \prime}(u, v)+0.5$. In Figure 2 , we see that $\pi_{G}$ decreases as $\lambda$ increases and the decreasing rate is more significant with large $c$. The presence of measurements errors can have a very substantial effect on $\pi_{G}$. For instance, for $c=1.5$ and $C_{p}^{\prime \prime}(u, v)=2.5, \pi_{G}$ is approximately equal to 1 without measurement errors and approximately equal to 0 when $\lambda=0.5$.

\section{Adjusted critical values}

As we have seen in the previous section, the $\alpha$-risk and the test power decrease with measurement errors. The capability testing results would be misleading if the producers do not take account of the gauge measurements errors. Thus, in order to improve the test power, we revise the critical value, denoted by $c_{0}^{A}$, to satisfy $c_{0}^{A}<c_{0}$. Let $\alpha_{A}=P\left(\hat{C}_{p}^{\prime \prime G}(u, v)>c_{0}^{A} \mid C_{p}^{\prime \prime}(u, v)=c\right)$ and $\pi_{A}\left(C_{p}^{\prime \prime}(u, v)\right)=P\left(\hat{C}_{p}^{\prime \prime G}(u, v)>c_{0}^{A} \mid C_{p}^{\prime \prime}(u, v)\right)$, be the $\alpha$-risk and the test power using the adjusted critical value. Since $c_{0}^{A}<c_{0}, P\left(\hat{C}_{p}^{\prime \prime G}(u, v)>c_{0}^{A}\right)$ is greater than $P\left(\hat{C}_{p}^{\prime \prime G}(u, v)>c_{0}\right)$, and both $\pi_{A}$ and $\alpha_{A}$ increase. To ensure that the $\alpha$-risk is within the preset magnitude, we set $\alpha_{A}=\alpha$, and solve equation $\alpha=P\left(\hat{C}_{p}^{\prime \prime G}(u, v)>c_{0}^{A} \mid C_{p}^{\prime \prime}(u, v)=c\right)$ to obtain $c_{0}^{A}$. Thus from (5), we must solve

$$
\alpha=\int_{0}^{K_{G}\left(c_{0}^{A}\right)} H_{G}\left(c_{0}^{A}, t\right) d t .
$$

Since the process parameters $\mu$ and $\sigma_{G}$ are unknown, then $\xi_{G}$ is also unknown. Thus the previous equation involves the unknown parameters $\xi_{G}$ and $C_{p}^{\prime \prime G}$ or $C_{p}^{\prime \prime}$ from (A.10). We replace $\xi_{G}$ by the observed value $\hat{\xi}_{G}$, and from (A.11),
$C_{p}^{\prime \prime}$ can be obtained by solving equation

$$
\begin{array}{r}
\frac{C_{p}^{\prime \prime}}{\sqrt{1+\lambda^{2} C_{p}^{\prime \prime 2} /(1-|\delta|)^{2}}}=\frac{\sqrt{1+v \hat{\xi}_{G}^{* 2}} \sqrt{1+v \hat{\xi}^{* 2}}}{\sqrt{1+\lambda^{2} C_{p}^{\prime \prime 2} /(1-|\delta|)^{2}+v \hat{\xi}^{* 2}}} \\
\times c+u(1-|\delta|) \hat{\xi}_{G}^{*} / 3,
\end{array}
$$

where $\hat{\xi}_{G}^{*}$ and $\hat{\xi}^{*}$ are defined from (A.3) and (A.14) as follows

$$
\hat{\xi}_{G}^{*}=\max \left((1-\delta)^{-1} \hat{\xi}_{G},-(1+\delta)^{-1} \hat{\xi}_{G}\right),
$$

and

$$
\begin{array}{r}
\hat{\xi}^{*}=\max \left(\frac{\hat{\xi}_{G}}{1-\delta} \sqrt{1+\lambda^{2}\left(\frac{\sqrt{1+v \hat{\xi}^{*^{2}}}}{1-|\delta|} c+u \hat{\xi}^{*} / 3\right)^{2}},\right. \\
\left.-\frac{\hat{\xi}_{G}}{1+\delta} \sqrt{1+\lambda^{2}\left(\frac{\sqrt{1+v \hat{\xi}^{*^{2}}}}{1-|\delta|} c+u \hat{\xi}^{*} / 3\right)^{2}}\right)
\end{array}
$$

With the adjusted critical value $c_{0}^{A}$, we can calculate the test power noted down as $\pi_{A}$. Let $C_{p 1}^{\prime \prime}$ be the value of $C_{p}^{\prime \prime}$ the solution of equation (6). The test power can be calculated as follows

$$
\begin{aligned}
& \pi_{A}\left(C_{p}^{\prime \prime}(u, v)\right)=P\left(\hat{C}_{p}^{\prime \prime G}(u, v)>c_{0}^{A} \mid C_{p}^{\prime \prime}(u, v), C_{p}^{\prime \prime}=C_{p 1}^{\prime \prime}\right)= \\
& \int_{0}^{K_{1}\left(c_{0}^{A}\right)} H_{1}\left(c_{0}^{A}, t\right) d t, \text { with } K_{1}(x)=b_{1} \sqrt{N} /((1-|\delta|) u+ \\
& 3 x \sqrt{v}), \\
& b_{1}=3 C_{p}^{\prime \prime G}=\frac{3 C_{p 1}^{\prime \prime}}{\sqrt{1+\lambda^{2} C_{p 1}^{\prime \prime 2} /(1-|\delta|)^{2}}} \text { from }(3), \\
& H_{1}(x, y)=F_{K}\left(\left(\frac{b_{1} \sqrt{N}-(1-|\delta|) u t}{3 x}\right)^{2}-v t^{2}\right) f_{G 1}(t),
\end{aligned}
$$

$f_{G 1}(t)=(1-\delta) \phi\left((1-\delta) t-\sqrt{N} \xi_{G 1}\right)+(1+\delta) \phi((1+\delta) t$ $\left.+\sqrt{N} \xi_{G 1}\right)$, and

$\xi_{G 1}=\left\{\begin{array}{l}(1-\delta) \xi_{G 1}^{*} \text { if } \xi_{G 1}>0 \\ -(1+\delta) \xi_{G 1}^{*} \text { if } \xi_{G 1}<0\end{array}\right.$ from (A.4).

$\xi_{1}^{*}$ then $\xi_{G 1}^{*}$ are obtained by substituting $C_{p 1}^{\prime \prime}$ to $C_{p}^{\prime \prime}$ in (A.15) and (A.13). $\xi_{G 1}$ and $\xi_{G}$ have the same sign, but $\xi_{G}$ is unknown. Thus we replace $\xi_{G}$ by the observed value $\hat{\xi}_{G}$, and replace $\xi_{G 1}$ by $\hat{\xi}_{G 1}$ defined by

$$
\hat{\xi}_{G 1}=\left\{\begin{array}{l}
(1-\delta) \xi_{G 1}^{*} \quad \text { if } \quad \hat{\xi}_{G}>0 \\
-(1+\delta) \xi_{G 1}^{*} \text { if } \quad \hat{\xi}_{G}<0
\end{array}\right.
$$

A Maple program is developed in Appendix B to compute the adjusted critical value $c_{0}^{A}$ and plot the power test. 


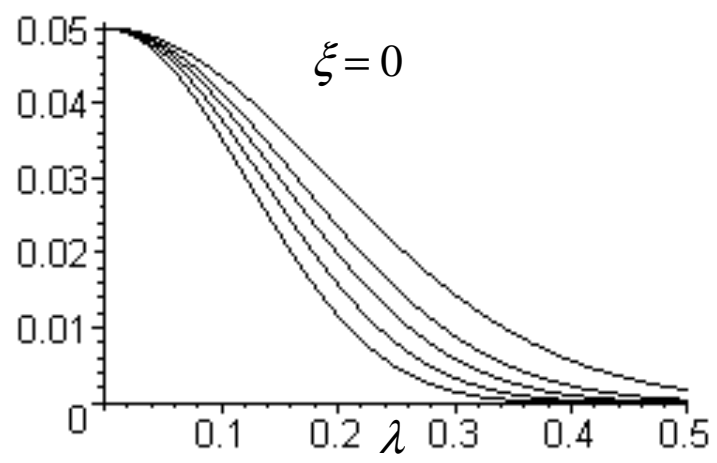

(a)
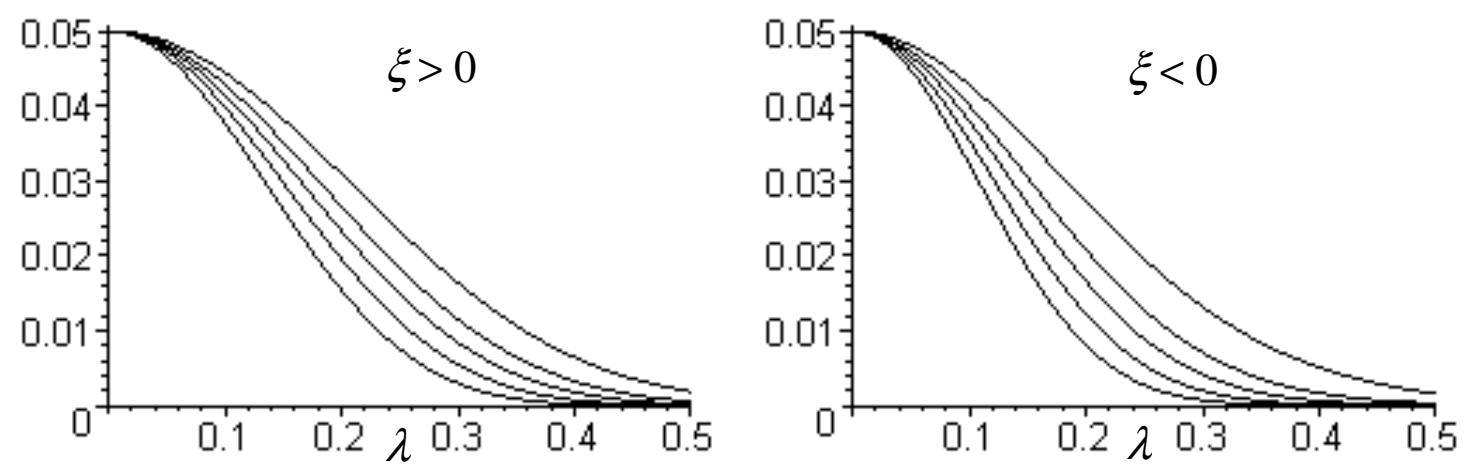

(b)
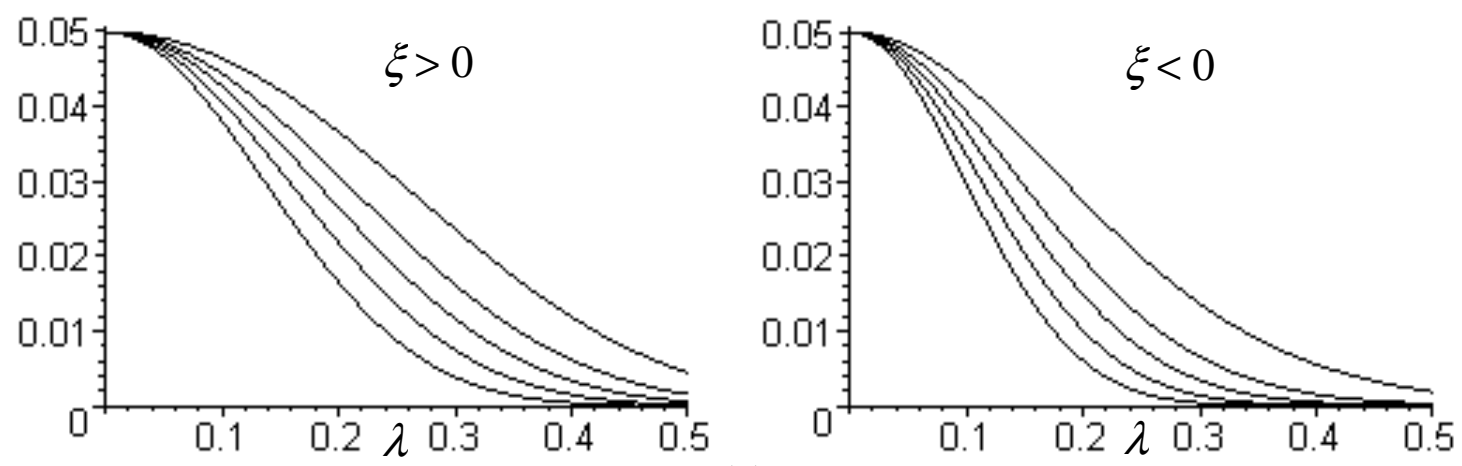

(c)

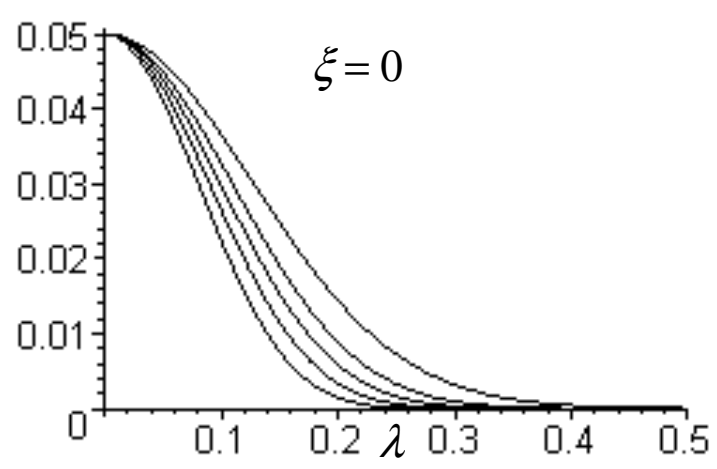

(d)

Fig. 1. Plots of $\alpha_{G}$ versus $\lambda$ with $r=1, N=30,50,70,100,150$ (top to bottom), $u=0.5, v=1.2, \delta=0.3, \alpha=0.05$ for (a) $c=1, C_{p}^{\prime \prime}=1$; (b) $c=1, C_{p}^{\prime \prime}=1.5$; (c) $c=1, C_{p}^{\prime \prime}=2$; (d) $c=1.5, C_{p}^{\prime \prime}=1.5$; (e) $c=1.5, C_{p}^{\prime \prime}=2$; (f) $c=1.5, C_{p}^{\prime \prime}=2.5$. 

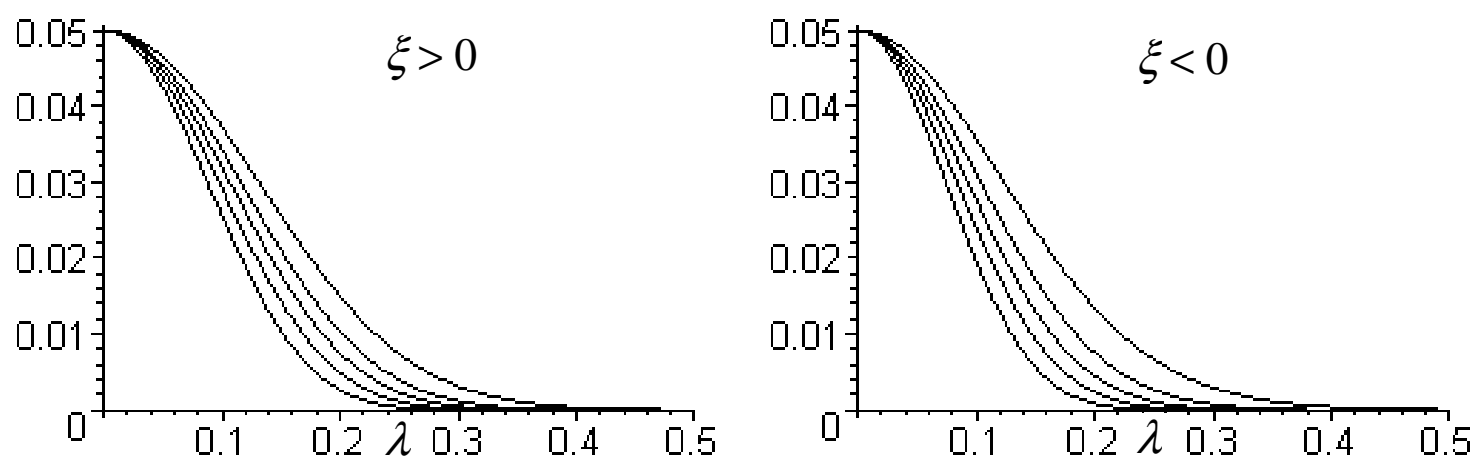

(e)
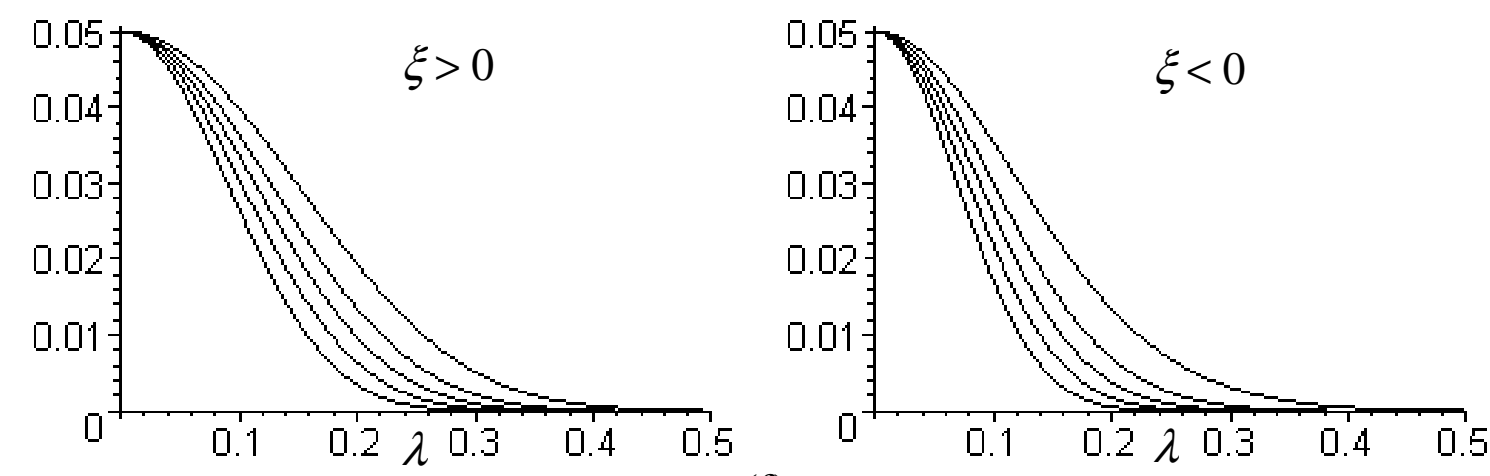

(f)

Fig. 1. Continued.

Figure 3 plots $\pi_{A}$ versus $\lambda \in[0,0.5]$ with $r=1, N=50$, $\alpha=0.05, c=1.00,1.50, C_{p}^{\prime \prime}(u, v)=c(0.20)(c+1)$ and $C_{p}^{\prime \prime}=C_{p}^{\prime \prime}(u, v), C_{p}^{\prime \prime}(u, v)+0.5$. In Figure $3, \pi_{A}$ decreases as increases and the decreasing rate is more significant with large $c$. However, if we compare Figures 2 and 3, we can see that the powers corresponding to the adjusted critical values $c_{0}^{A}$ decrease more slowly. Thus the test power is improved.

\section{Example}

The study we present here has been conducted within the company Chabert and Guillot producing nougat bars made with almonds, honey and other natural ingredients. At the output of the production, the nougat comes in the form of a $520 \mathrm{~mm}$ long block. A saw cuts this block into 40 of $13 \mathrm{~mm}$ bars, a weight sorter ejects the too heavy or too light bars, which are then recycled. Sets of about 10000 bars are then packaged. Bars weighing $200 \mathrm{~g}$ are sold. For a batch to be accepted, the French legislation requires that no bar should weigh less than $182 \mathrm{~g}$, there should not be more than $2 \%$ of bars between $182 \mathrm{~g}$ and $191 \mathrm{~g}$, and the mean weight should be at least $200 \mathrm{~g}$. Given the legislative and financial regulations, target $T$ has been set at $212 \mathrm{~g}$ and $L$ and $U$ tolerances at $191 \mathrm{~g}$ and $230 \mathrm{~g}$ (values beyond which the bars are recycled). We have $d^{*}=18$, and $\delta=0.077$. To determine the process capability, 20 blocks have been randomly selected. Due to a systematic deformation at the beginning and the end of the block, only 36 bars are used to determine the mean weight and the standard deviation of the weight of a bar. The data collected are presented in Table 1. Histogram plot shows that the collected data follow the normal distribution and the Shapiro-Wilk test is applied to further justify the assumption.

To choose an index $C_{p}^{\prime \prime}(u, v)$, Grau [11] suggests that it should be defined so that the process cannot be claimed as capable when the mean deviation and the proportion of non-conforming exceed the maximum limits. On the other hand, in order to have an easily interpretable measure, the process is said capable when $C_{p}^{\prime \prime}(u, v)$ is higher than or equal to 1 . For the firm, the process cannot be considered capable if the mean is less than $200 \mathrm{~g}$, that is to say if the mean moves away from more than $K=(T-\mu) D_{l}=57 \%$ of the distance between the target and the lower limit. Using the results Grau [11] obtained, for $u$ and $v$ values defined with a precision of 0.1 , Table 2 lists the pairs which get as close as possible to this requirement. This table also shows the maximum proportion $M$ of non-conforming bars when $C_{p}^{\prime \prime}(u, v)=1$. Given the annual costs of excessive dosage, the company has decided to use the $C_{p}^{\prime \prime}(0.8,0.1)$ index.

To determine whether the process is capable with a gauge capability $\lambda$ equal to 0.12 provided by a $R \& R$ 

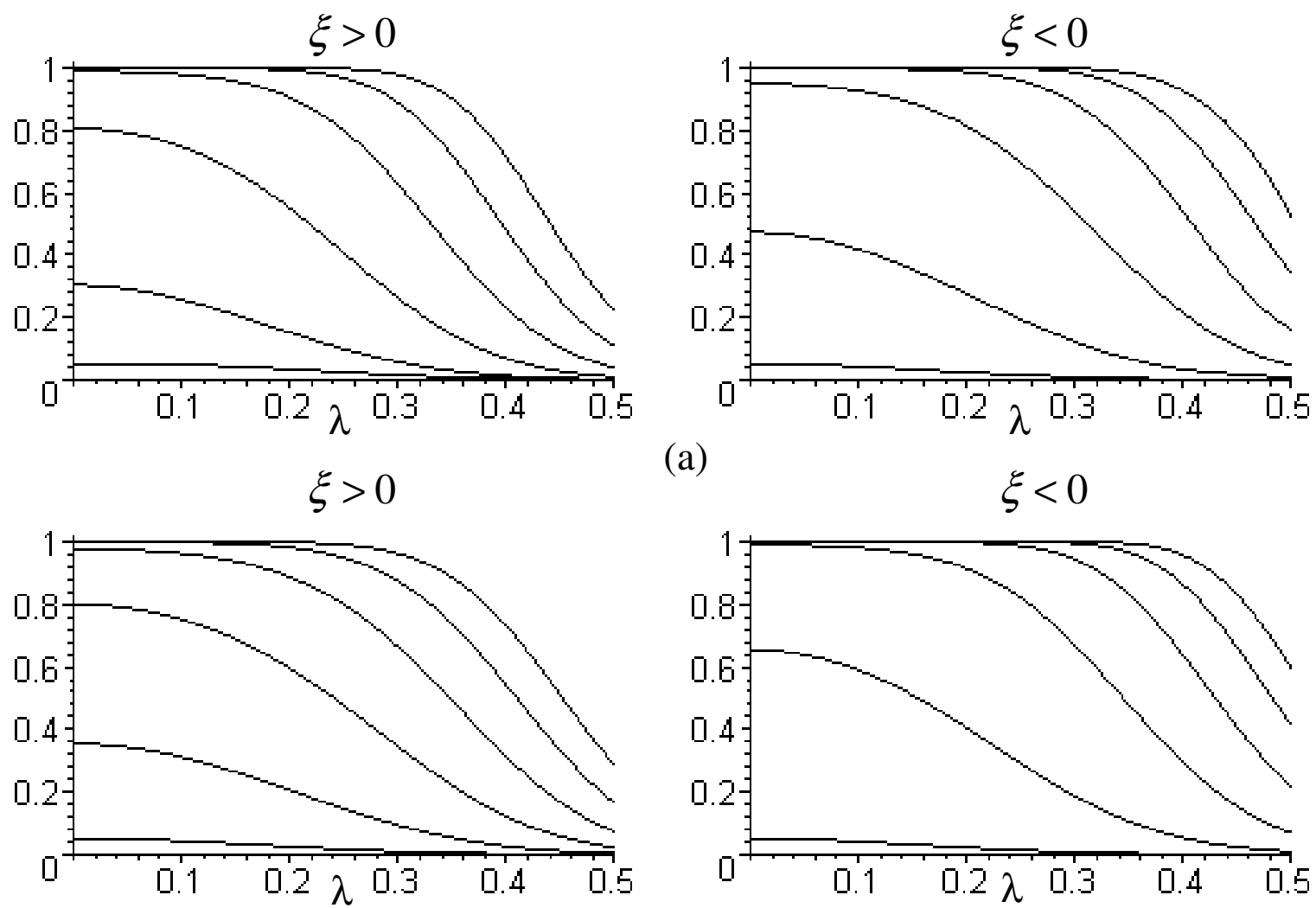

(a)

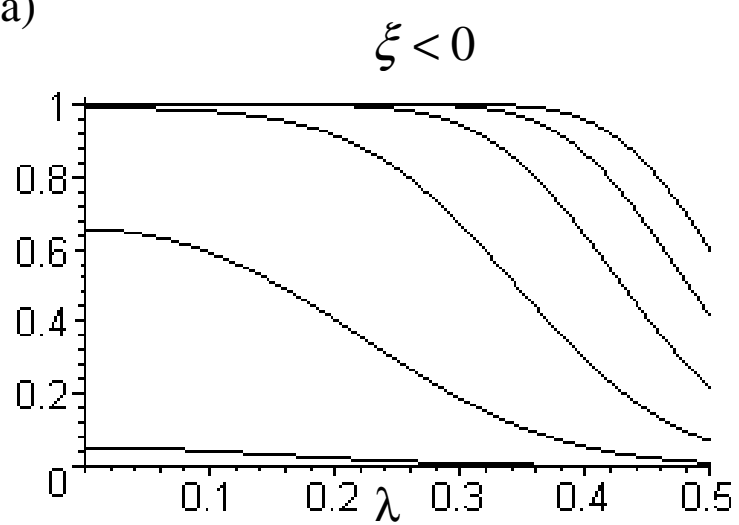

(b)
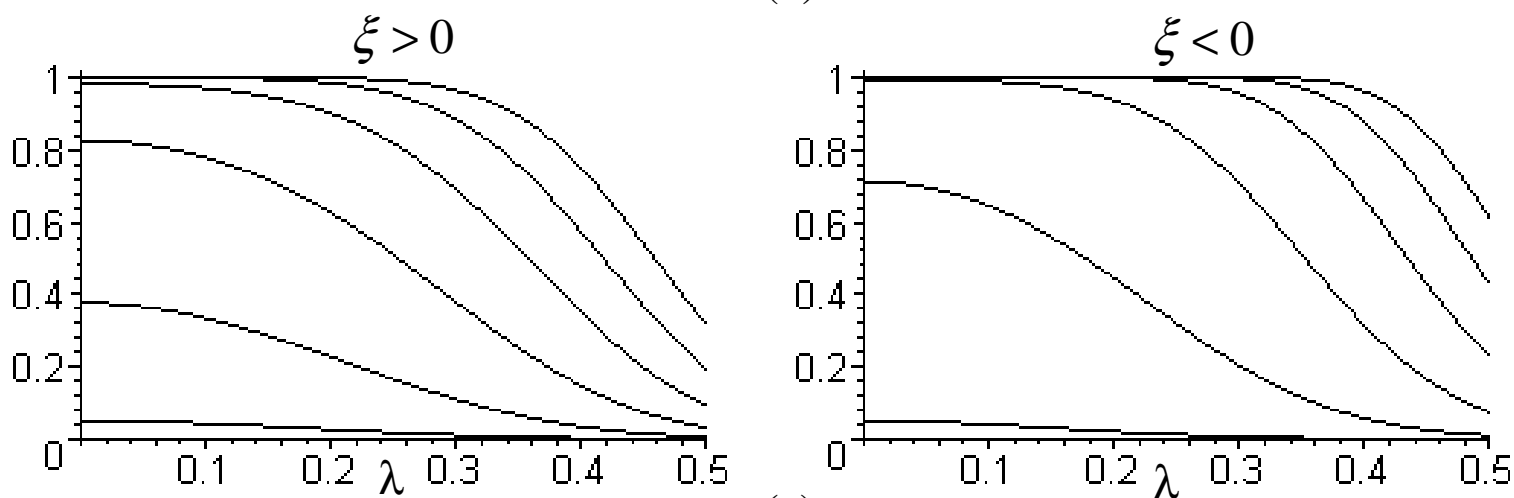

(c)
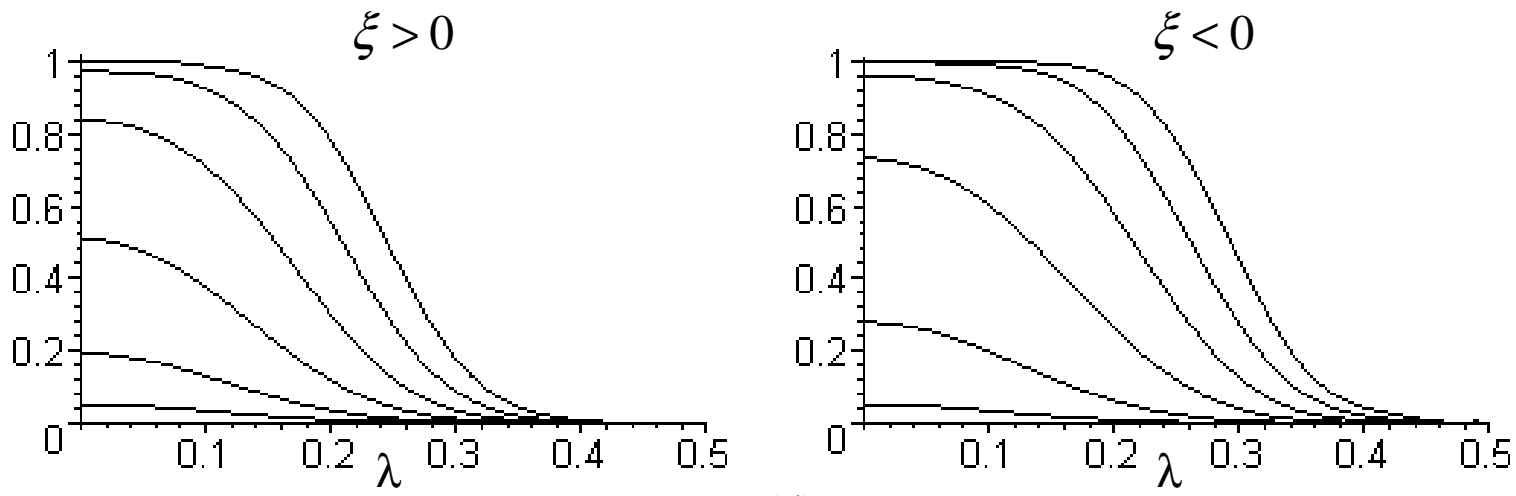

(d)

Fig. 2. Plots of $\pi_{G}$ versus $\lambda$ with $u=0.5, v=1.2, \delta=0.3, r=1, N=50, \alpha=0.05, C_{p}^{\prime \prime}(u, v)=c(0.20)(c+1)$ (bottom to top) for (a) $c=1$ and $C_{p}^{\prime \prime}=C_{p}^{\prime \prime}(u, v)$; (b) $c=1$ and $C_{p}^{\prime \prime}=C_{p}^{\prime \prime}(u, v)+0.33$; (c) $c=1$ and $C_{p}^{\prime \prime}=C_{p}^{\prime \prime}(u, v)+0.5$; (d) $c=1.5$ and $C_{p}^{\prime \prime}=C_{p}^{\prime \prime}(u, v) ;(\mathrm{e}) c=1.5$ and $C_{p}^{\prime \prime}=C_{p}^{\prime \prime}(u, v)+0.33$; (f) $c=1.5$ and $C_{p}^{\prime \prime}=C_{p}^{\prime \prime}(u, v)+0.5$. 


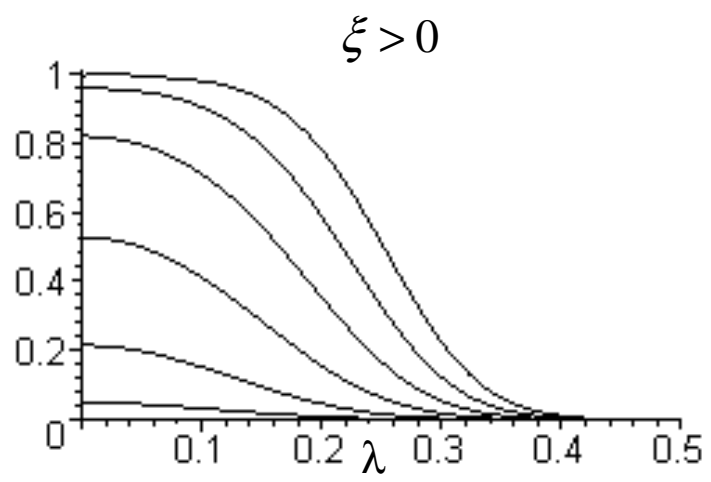

(e)
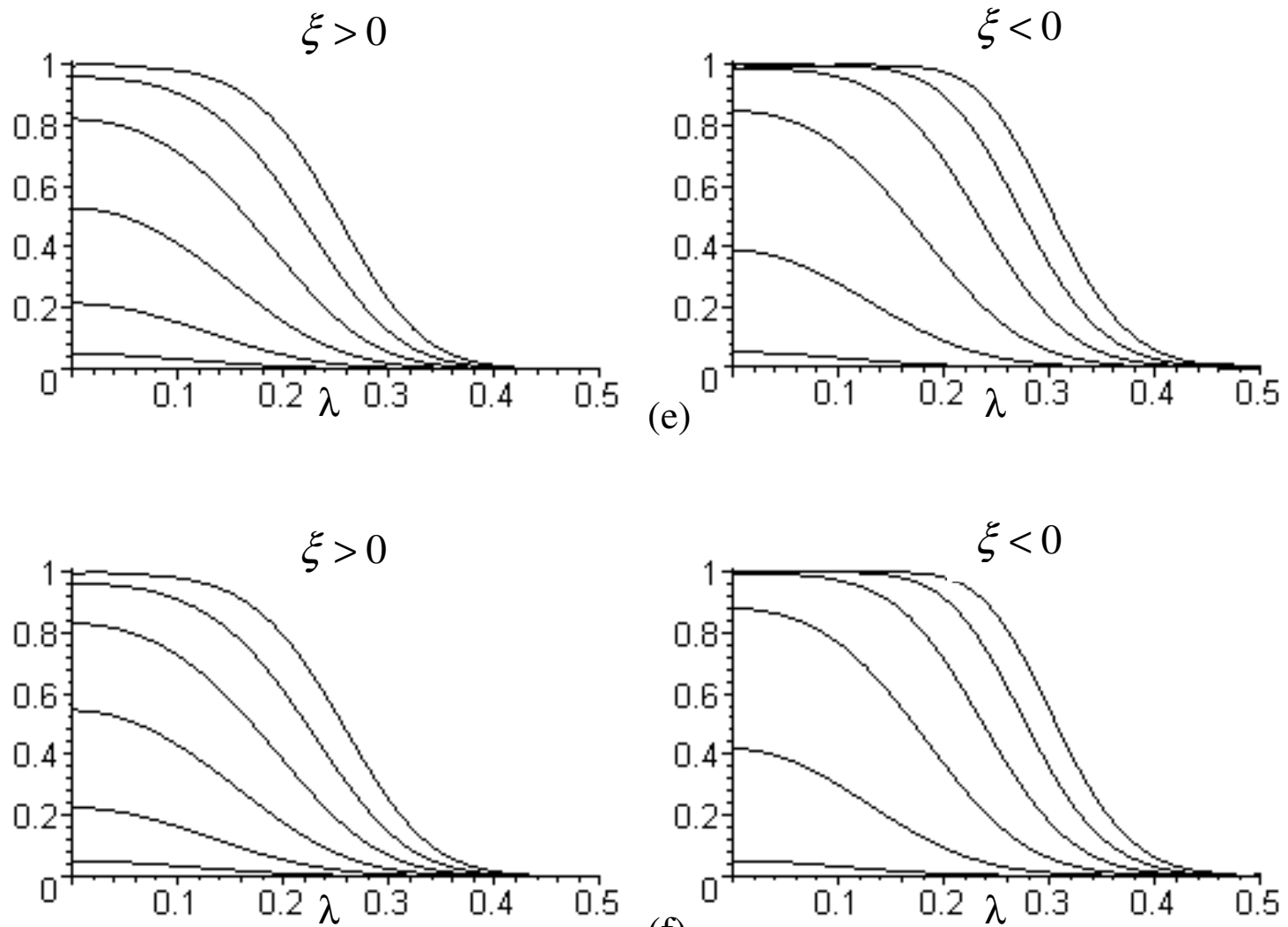

(f)

Fig. 2. Continued.

Table 1. Means and standard deviations of the 20 subsamples.

\begin{tabular}{cccccccc}
\hline Sample number & 1 & 2 & 3 & 4 & 5 & 6 & 7 \\
\hline $\bar{x}_{i}$ & 208.506 & 210.116 & 208.797 & 209.814 & 210.463 & 209.562 & 209.897 \\
$s_{i}$ & 4.712 & 3.648 & 3.264 & 3.437 & 4.418 & 4.518 & 4.225 \\
\hline Sample number & 8 & 9 & 10 & 11 & 12 & 13 & 14 \\
$\bar{x}_{i}$ & 209.553 & 209.286 & 209.577 & 210.851 & 210.168 & 211.011 & 211.018 \\
$s_{i}$ & 4.632 & 4.825 & 4.654 & 4.365 & 4.153 & 4.419 & 5.170 \\
\hline Sample number & 15 & 16 & 17 & 18 & 19 & 20 & \\
$\bar{x}_{i}$ & 209.464 & 210.554 & 209.582 & 209.776 & 211.002 & 210.882 & \\
$s_{i}$ & 4.709 & 4.695 & 4.258 & 4.943 & 4.834 & 3.946 & \\
\hline
\end{tabular}

Table 2. Pairs $(u, v)$ for which $K$ is roughly equal to $57 \%$ when $C_{p}^{\prime \prime}(u, v)=1$, and $M$ the maximum percentage of nonconforming.

\begin{tabular}{ccccccc}
\hline$(u, v)$ & $(0,0.3)$ & $(0.1,0.3)$ & $(0.3,0.2)$ & $(0.4,0.2)$ & $(0.7,0.1)$ & $(0.8,0.1)$ \\
\hline$K$ & $56 \%$ & $53 \%$ & $57 \%$ & $54 \%$ & $58 \%$ & $55 \%$ \\
$M$ in ppm & 6532 & 4821 & 6127 & 3143 & 2057 & 1628 \\
\hline
\end{tabular}

analysis, we determine that $c=1$ and $\alpha=0.05$. Then, based on the sample data of $N=720$ observations, we obtain $\overline{\bar{G}}=209.994, S_{G}=4.418, \hat{\xi}_{G}=-0.454$ and $\hat{c}_{p}^{\prime \prime G}(0.8,0.1)=1.282$. From the Maple program in Appendix $\mathrm{B}$, we obtain the critical value $c_{0}^{A}=1.052$. Since $\hat{c}_{p}^{\prime \prime G}(0.5,0.1)>c_{0}^{A}$, we can conclude that the process is capable. We also see that if we ignore the measurements errors, the critical value may be calculated as $c_{0}=1.063$. So if another sample gives $\overline{\bar{G}}=209.590$ and $S_{G}=5.307$, we always have $\hat{\xi}_{G}=-0.454$, thus $c_{0}^{A}=1.052$ and $c_{0}=1.063$. Since $\hat{c}_{p}^{\prime \prime G}(0.8,0.1)=1.056$, we conclude that the process is capable, but we cannot draw the same conclusion if we ignore the measurement errors.

\section{Conclusion}

In this work we have been concerned with the problem of gauge measurement errors when dealing with process 


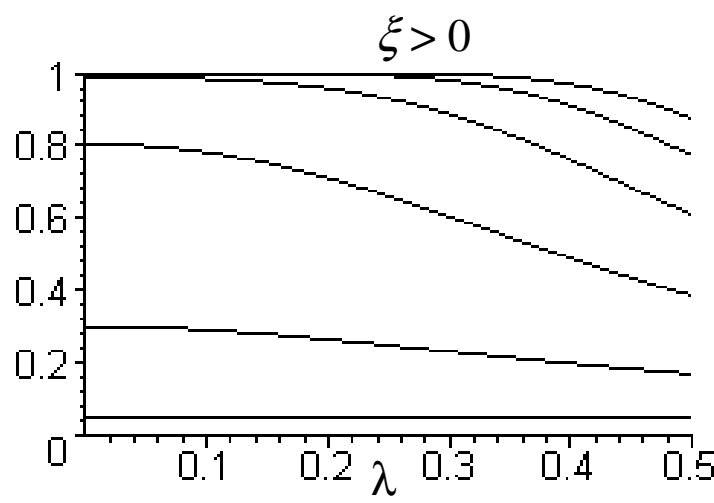

(a)
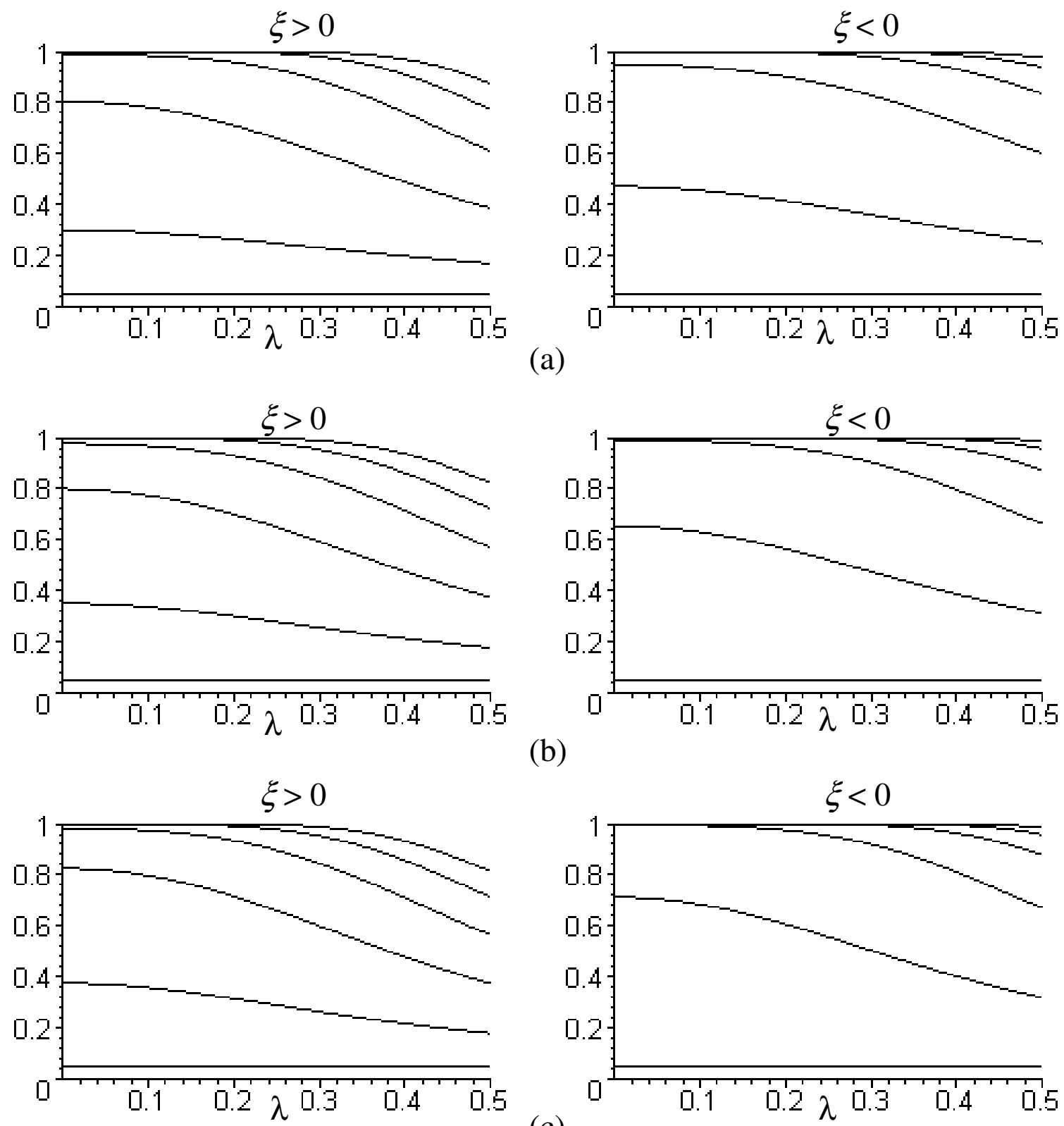

(b)

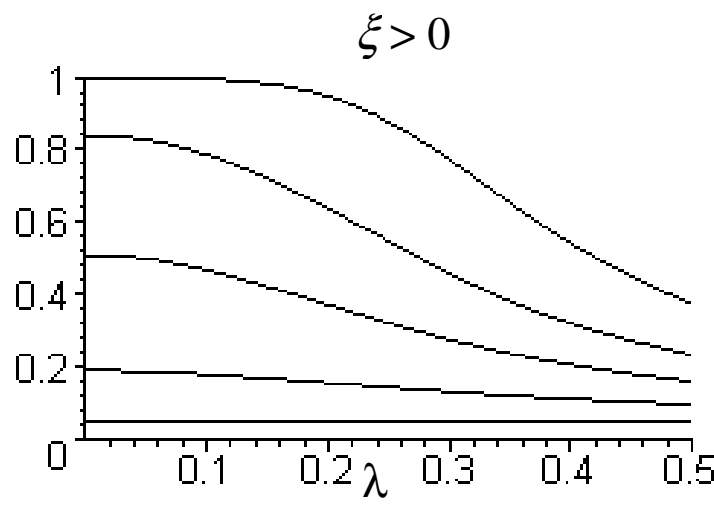

(c)

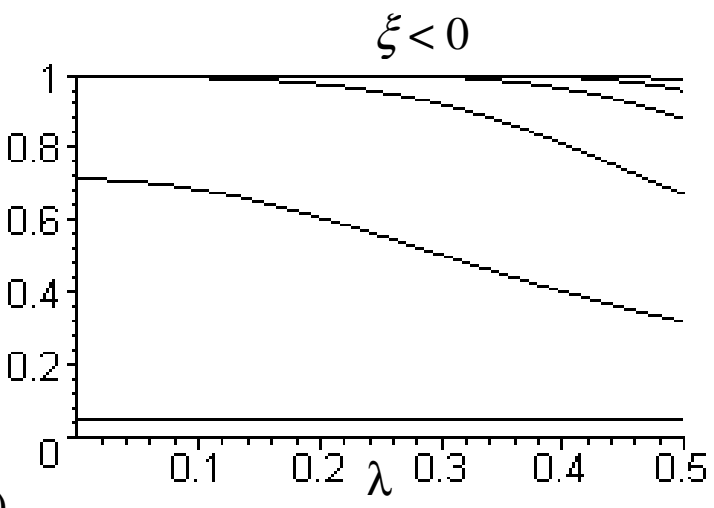

(d)

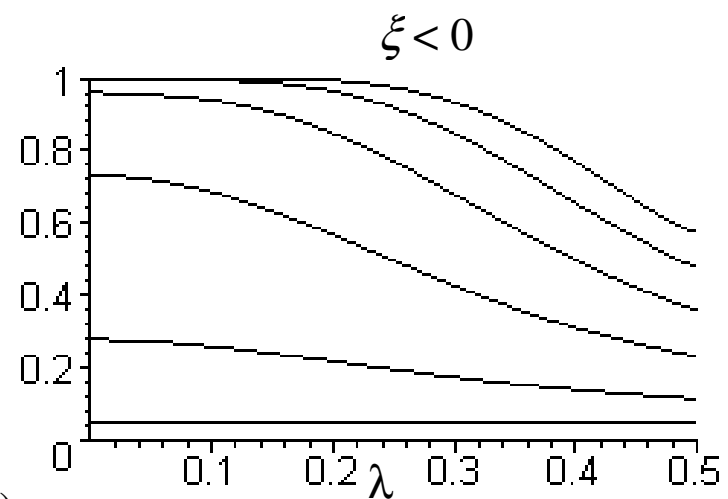

Fig. 3. Plots of $\pi_{A}$ versus with $u=0.5, v=1.2, \delta=0.3, r=1, N=50, \alpha=0.05, C_{p}^{\prime \prime}(u, v)=c(0.20)(c+1)$ (bottom to top) for (a) $c=1$ and $C_{p}^{\prime \prime}=C_{p}^{\prime \prime}(u, v)$; (b) $c=1$ and $C_{p}^{\prime \prime}=C_{p}^{\prime \prime}(u, v)+0.33$; (c) $c=1$ and $C_{p}^{\prime \prime}=C_{p}^{\prime \prime}(u, v)+0.5 ;(\mathrm{d}) c=1.5$ and $C_{p}^{\prime \prime}=C_{p}^{\prime \prime}(u, v) ;(\mathrm{e}) c=1.5$ and $C_{p}^{\prime \prime}=C_{p}^{\prime \prime}(u, v)+0.33 ;$ (f) $c=1.5$ and $C_{p}^{\prime \prime}=C_{p}^{\prime \prime}(u, v)+0.5$. 

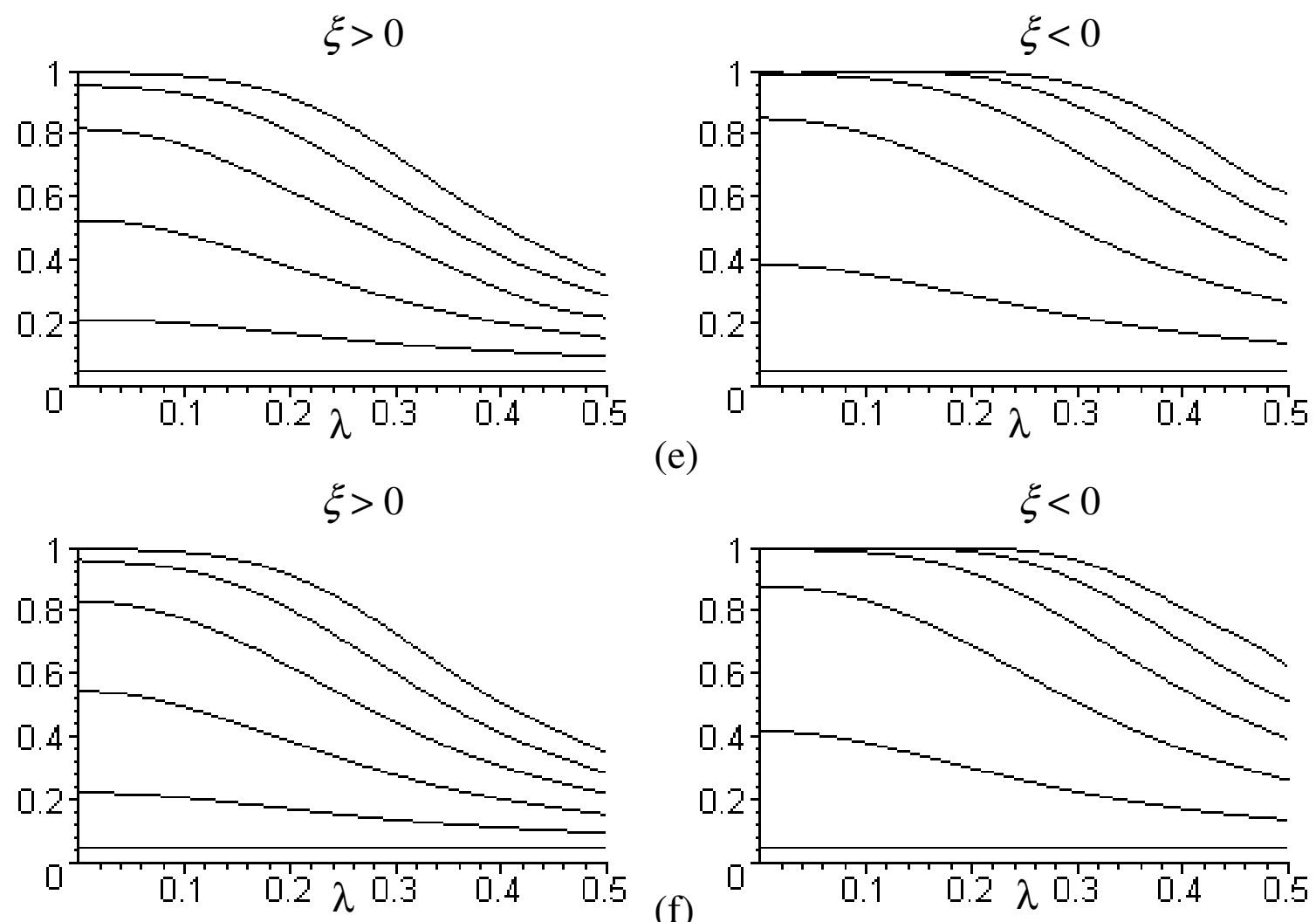

(e)

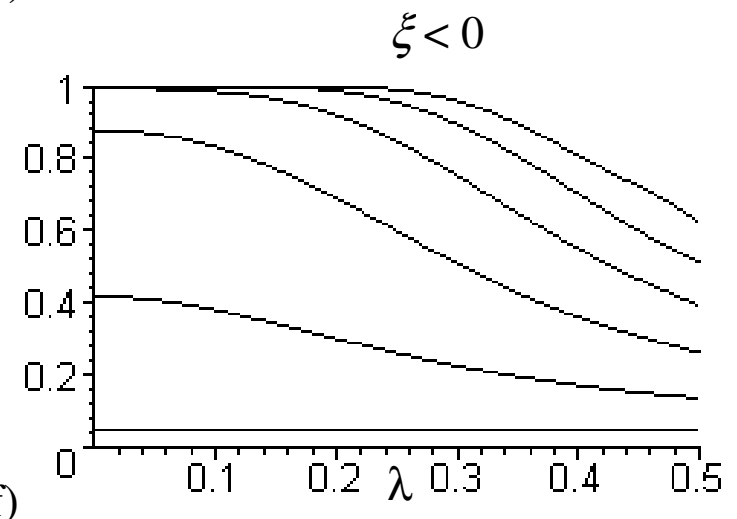

Fig. 3. Continued.

capability indices for symmetric or asymmetric tolerances. Gauge measurement errors have a significant impact on the estimation of a process capability. When small subsamples data are collected from past "in-control", the probability distribution of the estimators of the process capability $C_{p}^{\prime \prime}(u, v)$ and the process capability with gauge measurement errors $C_{p}^{\prime \prime} G(u, v)$ has been investigated to obtain critical values and power test. The estimator evaluating the capability by using the sample data contaminated with gauge measurement errors severely underestimates the $\alpha$-risk, resulting in a smaller testing power. We suggest an adjusted critical value to improve the accuracy of the capability assessment. For practical purpose we give a Maple program helpful to the users for their factory applications. Finally a real example in a company producing nougat is used to assess the interest of the approach.

\section{Appendix A}

- We have

$$
\begin{gathered}
\xi^{*}=\max \left((1-\delta)^{-1} \xi,-(1+\delta)^{-1} \xi\right) \\
\xi=\left\{\begin{array}{lll}
(1-\delta) \xi^{*} & \text { if } \quad \xi>0 \\
-(1+\delta) \xi^{*} & \text { if } \quad \xi<0
\end{array}\right. \\
\xi_{G}^{*}=\max \left((1-\delta)^{-1} \xi_{G},-(1+\delta)^{-1} \xi_{G}\right) \\
\xi_{G}=\left\{\begin{array}{lll}
(1-\delta) \xi_{G}^{*} & \text { if } \quad \xi_{G}>0 \\
-(1+\delta) \xi_{G}^{*} & \text { if } \quad \xi_{G}<0
\end{array}\right.
\end{gathered}
$$

- Since $C_{p}=C_{p}(0,0)$ and $C_{p}^{\prime \prime}=C_{p}^{\prime \prime}(0,0)$, we have

$$
C_{p}=C_{p}^{\prime \prime} /(1-|\delta|) .
$$

- Since $\xi^{*}=A / \sigma$ and $A^{*}=(1-|\delta|) A$, from (1) we have $C_{p}^{\prime \prime}(u, v)=\frac{C_{p}^{\prime \prime}-u A^{*} /(3 \sigma)}{\sqrt{1+v A^{2} / \sigma^{2}}}=\frac{C_{p}^{\prime \prime}-u(1-|\delta|) \xi^{*} / 3}{\sqrt{1+v \xi^{* 2}}}$, thus

$$
C_{p}^{\prime \prime}=\sqrt{1+v \xi^{* 2}} C_{p}^{\prime \prime}(u, v)+u(1-|\delta|) \xi^{*} / 3 .
$$

- In the same manner, from (2) we have

$$
C_{p}^{\prime \prime G}=\sqrt{1+v \xi_{G}^{*^{2}}} C_{p}^{\prime \prime G}(u, v)+u(1-|\delta|) \xi_{G}^{*} / 3 .
$$

- From (A.5) and (A.6) we have

$$
C_{p}=\sqrt{1+v \xi^{*^{2}}} C_{p}^{\prime \prime}(u, v) /(1-|\delta|)+u \xi^{*} / 3 .
$$

- From (A.7) and (3), we have

$$
\begin{aligned}
C_{p}^{\prime \prime G}= & \frac{\sqrt{1+v \xi_{G}^{*^{2}}} \sqrt{1+v \xi^{* 2}}}{\sqrt{1+\lambda^{2} C_{p}^{\prime \prime 2} /(1-|\delta|)^{2}+v \xi^{* 2}}} \\
& \times C_{p}^{\prime \prime}(u, v)+u(1-|\delta|) \xi_{G}^{*} / 3 .
\end{aligned}
$$

- Since $C_{p}^{\prime \prime G}=C_{p}^{\prime \prime G}(0,0)$, from (3) we have

$$
C_{p}^{\prime \prime G}=C_{p}^{\prime \prime} / \sqrt{1+\lambda^{2} C_{p}^{\prime \prime 2} /(1-|\delta|)^{2}} .
$$




$$
\begin{aligned}
\xi^{*} & =\frac{-C_{0} u(1-|\delta|) / 3 \pm \sqrt{C^{2}\left(v\left(C_{0}^{2}-C^{2}\right)+(u(1-|\delta|) / 3)^{2}\right)}}{v C^{2}-(u(1-|\delta|) / 3)^{2}} \\
& =\frac{-C_{0} u(1-|\delta|) / 3 \pm \sqrt{C_{0}^{2}(u(1-|\delta|) / 3)^{2}+\left(C_{0}^{2}-C^{2}\right)\left(v C^{2}-(u(1-|\delta|) / 3)^{2}\right)}}{v C^{2}-(u(1-|\delta|) / 3)^{2}} \\
& =\frac{-a \pm \sqrt{a^{2}+\alpha b}}{b}
\end{aligned}
$$

- From (A.10) and (A.9), we have

$$
\begin{aligned}
& \frac{C_{p}^{\prime \prime}}{\sqrt{1+\lambda^{2} C_{p}^{\prime \prime 2} /(1-|\delta|)^{2}}}= \\
& \frac{\sqrt{1+v \xi_{G}^{*^{2}}} \sqrt{1+v \xi^{* 2}}}{\sqrt{1+\lambda^{2} C_{p}^{\prime \prime 2} /(1-|\delta|)^{2}+v \xi^{* 2}}} C_{p}^{\prime \prime}(u, v) \\
& +u(1-|\delta|) \xi_{G}^{*} / 3 .
\end{aligned}
$$

- Since $\xi=(\mu-T) / \sigma$ and $\xi_{G}=(\mu-T) / \sigma_{G}$, we have $\xi_{G}=\xi \sigma / \sigma_{G}=\xi \sigma / \sqrt{\sigma^{2}+\sigma_{M}^{2}}=$ $\xi / \sqrt{1+\sigma_{M}^{2} / \sigma^{2}}=\xi / \sqrt{1+\lambda^{2} C_{p}^{2}}$, and from (A.5) we have

$$
\xi_{G}=\xi / \sqrt{1+\lambda^{2} C_{p}^{\prime \prime 2} /(1-|\delta|)^{2}} .
$$

- From (A.3), (A.12) and (A.1), we have

$$
\xi_{G}^{*}=\xi^{*} / \sqrt{1+\lambda^{2} C_{p}^{\prime \prime 2} /(1-|\delta|)^{2}} .
$$

- From (A.1), (A.12), (A.5) and (A.8), we have

$$
\begin{gathered}
\xi^{*}=\max \left(\frac{\xi_{G}}{1-\delta} \sqrt{1+\lambda^{2}\left(\frac{\sqrt{1+v \xi^{*^{2}}}}{1-|\delta|} C_{p}^{\prime \prime}(u, v)+u \xi^{*} / 3\right)^{2}}\right. \\
-\frac{\xi_{G}}{1+\delta} \sqrt{\left.1+\lambda^{2}\left(\frac{\sqrt{1+v \xi^{*^{2}}}}{1-\delta \mid} C_{p}^{\prime \prime}(u, v)+u \xi^{*} / 3\right)^{2}\right)} . \quad \text { (A.14) }
\end{gathered}
$$

- We have $C_{p}^{\prime \prime}(u, v)=\frac{d^{*}-u A^{*}}{3 \sqrt{\sigma^{2}+v A^{2}}}=\frac{C_{p}^{\prime \prime}-u A^{*} /(3 \sigma)}{\sqrt{1+v A^{2} / \sigma^{2}}}=$ $\frac{C_{p}^{\prime \prime}-u(1-|\delta|) \xi^{*} / 3}{\sqrt{1+v \xi^{* 2}}}$

$$
\Leftrightarrow\left\{\begin{array}{l}
\left(C_{p}^{\prime \prime}(u, v)\right)^{2}\left(1+v \xi^{* 2}\right)=\left(C_{p}^{\prime \prime}-u \xi^{*}(1-|\delta|) / 3\right)^{2} \text { (a) } \\
\xi^{*}<3 C_{p}^{\prime \prime} /(u(1-|\delta|))
\end{array}\right.
$$

Assume that $C=C_{p}^{\prime \prime}(u, v)$ and $C_{0}=C_{p}^{\prime \prime}$.

$$
\begin{array}{r}
(\mathrm{a}) \Leftrightarrow C^{2}+v C^{2} \xi^{* 2}=C_{0}^{2}+\xi^{* 2}(u(1-|\delta|) / 3)^{2} \\
-2 C_{0} u \xi^{*}(1-|\delta|) / 3 \\
\Leftrightarrow\left(v C^{2}-(u(1-|\delta|) / 3)^{2}\right) \xi^{* 2}+2 C_{0} u \xi^{*}(1-|\delta|) / 3 \\
+C^{2}-C_{0}^{2}=0,
\end{array}
$$

which is a second-degree polynomial of the variable $\xi^{*}$, and the discriminant is

$$
\begin{aligned}
\Delta^{\prime} & =\left(C_{0} u(1-|\delta|) / 3\right)^{2}-\left(v C^{2}-(u(1-|\delta|) / 3)^{2}\right) \\
& \times\left(C^{2}-C_{0}^{2}\right) \\
& =\left(v C^{2}\left(C_{0}^{2}-C^{2}\right)+C^{2}(u(1-|\delta|) / 3)^{2}\right) \\
& =C^{2}\left(v\left(C_{0}^{2}-C^{2}\right)+(u(1-|\delta|) / 3)^{2}\right) .
\end{aligned}
$$

Then $\alpha=C_{0}^{2}-C^{2}=\left(C_{p}^{\prime \prime}\right)^{2}-\left(C_{p}^{\prime \prime}(u, v)\right)^{2} \geqslant 0$, thus $\Delta^{\prime} \geqslant 0$, and

\section{See Equation above.}

with $a=C_{0} u(1-|\delta|) / 3 \geqslant 0$ and $b=v C^{2}-(u(1-|\delta|) / 3)^{2}$.

Thus the solutions of the equation are $\xi_{1}^{*}=\frac{-a+\sqrt{a^{2}+\alpha b}}{b}$ and $\xi_{2}^{*}=\frac{-a-\sqrt{a^{2}+\alpha b}}{b}$. However $\xi^{*}=\max \left((1-\delta)^{-1} \xi,-(1+\right.$ $\left.\delta)^{-1} \xi\right)$ must necessarily be positive or equal to zero.

(1) If $C=C_{p}^{\prime \prime}(u, v)>\frac{u(1-|\delta|)}{3 \sqrt{v}}$, then $b>0$ and $\xi_{2}^{*}<0$ is not suitable.

(2) If $0<C=C_{p}^{\prime \prime}(u, v)<\frac{u(1-|\delta|)}{3 \sqrt{v}}$, then

$0<v C^{2}<(u(1-|\delta|) / 3)^{2} \Leftrightarrow-(u(1-|\delta|) / 3)^{2}<b<0$. Thus

$$
a^{2}-\alpha(u(1-|\delta|) / 3)^{2}<a^{2}+\alpha b
$$

$\Leftrightarrow C_{0}^{2}(u(1-|\delta|) / 3)^{2}-\left(C_{0}^{2}-C^{2}\right)(u(1-|\delta|) / 3)^{2}<a^{2}+\alpha b$

$\Leftrightarrow C^{2}(u(1-|\delta|) / 3)^{2}<a^{2}+\alpha b \Leftrightarrow C(u(1-|\delta|) / 3)<$ $\sqrt{a^{2}+\alpha b} \Leftrightarrow \frac{-a-C u(1-|\delta|) / 3}{b}<\frac{-a-\sqrt{a^{2}+\alpha b}}{b}$, since $b<0$

$$
\begin{gathered}
\Leftrightarrow \frac{-a-C u(1-|\delta|) / 3}{v C^{2}-(u(1-|\delta|) / 3)^{2}}<\xi_{2}^{*} \Leftrightarrow \frac{a+C u(1-|\delta|) / 3}{(u(1-|\delta|) / 3)^{2}-v C^{2}}<\xi_{2}^{*} \\
\Leftrightarrow \frac{a+C u(1-|\delta|) / 3}{(u(1-|\delta|) / 3)^{2}}<\xi_{2}^{*} \Leftrightarrow \frac{a}{(u(1-|\delta|) / 3)^{2}}<\xi_{2}^{*} \\
\Leftrightarrow \frac{3}{u(1-|\delta|)} C_{0}<\xi_{2}^{*}
\end{gathered}
$$

$\Leftrightarrow \xi_{2}^{*}>3 C_{p}^{\prime \prime G} /(u(1-|\delta|))$, is a solution that does not check (b), thus it is not suitable. Therefore the only possible solution is $\xi^{*}=\xi_{1}^{*}=\frac{-a+\sqrt{a^{2}+\alpha b}}{b}$, that is to say 


$$
\xi^{*}=\frac{-C_{p}^{\prime \prime} u(1-|\delta|) / 3+\sqrt{\left(C_{p}^{\prime \prime}\right)^{2}(u(1-|\delta|) / 3)^{2}+\left(\left(C_{p}^{\prime \prime}\right)^{2}-\left(C_{p}^{\prime \prime}(u, v)\right)^{2}\right)\left(v\left(C_{p}^{\prime \prime}(u, v)\right)^{2}-(u(1-|\delta|) / 3)^{2}\right)}}{v\left(C_{p}^{\prime \prime}(u, v)\right)^{2}-(u(1-|\delta|) / 3)^{2}}
$$

See equation (A.15) above.

\section{Appendix B}

When the function $H_{G}$ takes values that are too low, the Maple software is sometimes unable to perform the calculation of the integral of $H_{G}$. This is the reason why we limit the domain of integration $\left[0 ; K_{G}\right]$ to the domain $[K 0 ; K 1]$ on which $H_{G}>10^{-8}$. This does not change the value of the integral for the required precision.

Maple Program to compute the adjusted critical value $c_{0}^{A}$ and the power test.

\section{Algorithm}

Step 1. Read $u, v, \delta, \lambda, r, N, \alpha, c, \hat{\xi}_{G}$

Step 2. Compute $\hat{\xi}^{*}$ from (8), $\hat{\xi}_{G}^{*}$ from (7), $C_{p}^{\prime \prime}$ from (6), $C_{p}^{\prime \prime G}$ from (A.10).

Step 3. Compute $H_{G}, K_{G}$.

Step 4. Find the adjusted critical value $c_{0}^{A}$.

Step 4.1. Find $K 0$ a lower integration bound.

Step 4.2. Find $K 1$ an upper integration bound.

Step 5. Print the adjusted critical value.

Step 6: Plot the power test.

Maple program restart:with(stats):

\#1. Read u, v, delta, lambda, r (sub-samples number), $\mathrm{N}$ (total number of observations), alpha (the alpha-risk), c (threshold value for a capable process), $\mathrm{kG}$ (estimation of (muG-T)/sigmaG), cpuvG (estimation of $\mathrm{C}^{\prime \prime}$ puvG, to obtain the power test)

$\mathrm{u}:=0.8: \mathrm{v}:=0.1:$ delta: $=0.077:$ lambda: $=0.12$ :

$\mathrm{r}:=20: \mathrm{N}:=720$ :alpha: $=0.05: \mathrm{c}:=1: \mathrm{kG}:=-0.454$ :

\#2. Compute ks (estimation of xi_star), kGs (estimation of xiG_star), $\mathrm{Cp}$ ( $\left.\mathrm{C}^{\prime \prime} \mathrm{p}\right), \mathrm{CpG}\left(\mathrm{C}^{\prime \prime} \mathrm{pG}\right)$

$\mathrm{ks}:=\operatorname{solve}(\mathrm{x}=$ piecewise $(\mathrm{kG}>0, \mathrm{kG} /(1-$

delta $)^{*} \operatorname{sqrt}\left(1+\operatorname{lambda} 2^{*}\left(\operatorname{sqrt}\left(1+\mathrm{v}^{*} \mathrm{x}^{\wedge} 2\right) /\right.\right.$

$\left.\left.(1-\operatorname{abs}(\text { delta }))^{*} \mathrm{c}+\mathrm{u}^{*} \mathrm{x} / 3\right)^{\wedge} 2\right)$,-

$\mathrm{kG} /(1+$ delta $) * \operatorname{sqrt}\left(1+\operatorname{lambda}^{\wedge} 2^{*}\left(\operatorname{sqrt}\left(1+\mathrm{v}^{*} \mathrm{x}^{\wedge} 2\right) /(1-\right.\right.$

$\operatorname{abs}($ delta $\left.\left.\left.\left.))^{*} \mathrm{c}+\mathrm{u}^{*} \mathrm{x} / 3\right)^{\wedge} 2\right)\right), \mathrm{x}\right)$ :

$\mathrm{kGs}:=\max (\mathrm{kG} /(1$-delta $),-\mathrm{kG} /(1+$ delta $))$ :

$\mathrm{Cp}:=\operatorname{fsolve}\left(\mathrm{x} / \operatorname{sqrt}\left(1+\operatorname{lambda} 2^{*} \mathrm{x}^{\wedge} 2 /(1-\operatorname{abs}(\text { delta }))^{\wedge} 2\right)=\right.$ $\operatorname{sqrt}\left(1+\mathrm{v}^{*} \mathrm{kGs}^{\wedge} 2\right) * \operatorname{sqrt}(1$

$\left.+\mathrm{v}^{*} \mathrm{ks}^{\wedge} 2\right)^{*} \mathrm{c} / \operatorname{sqrt}\left(1+\operatorname{lambda} 2^{*} \mathrm{x}^{\wedge} 2 /(1-\operatorname{abs}(\text { delta }))^{\wedge} 2\right.$

$\left.\left.+\mathrm{v}^{*} \mathrm{ks}^{\wedge} 2\right)+\mathrm{u}^{*}(1-\operatorname{abs}(\text { delta }))^{*} \mathrm{kGs} / 3, \mathrm{x}=0 . .10\right)$ :

$\mathrm{CpG}:=\mathrm{Cp} / \operatorname{sqrt}\left(1+\operatorname{lambda}^{\wedge} 2^{*} \mathrm{Cp}^{\wedge} 2 /(1-\operatorname{abs}(\text { delta }))^{\wedge} 2\right)$ :

\#3. Compute H (HG) and K (KG)

$\mathrm{F}:=(\mathrm{c} 0, \mathrm{t})->$ stats [statevalf,cdf, chisquare[N-

$\mathrm{r}]]\left(\left(3^{*} \mathrm{CpG}^{*} \operatorname{sqrt}(\mathrm{N})-(1-\operatorname{abs}(\text { delta }))^{*} \mathrm{u}^{*} \mathrm{t}\right)^{\wedge} 2 / 9 / \mathrm{c}^{\wedge} 2-\right.$ $\left.\mathrm{v}^{*} \mathrm{t}^{\wedge} 2\right)$ : $\mathrm{f}:=\mathrm{t}->(1$-delta $) *$ stats[statevalf,pdf,normald] ((1-delta)*t$\left.\mathrm{kG}^{*} \mathrm{~N}^{\wedge} .5\right)+(1+$ delta $)$

${ }^{*}$ stats [statevalf,pdf,normald] $\left((1+\right.$ delta $\left.) * \mathrm{t}+\mathrm{kG}^{*} \mathrm{~N}^{\wedge} .5\right)$ :

$\mathrm{H}:=(\mathrm{c} 0, \mathrm{t})->\mathrm{F}(\mathrm{c} 0, \mathrm{t})^{*} \mathrm{f}(\mathrm{t})$ :

$\mathrm{K}:=3^{*} \mathrm{CpG}^{*} \operatorname{sqrt}(\mathrm{N}) /\left((1-\operatorname{abs}(\text { delta }))^{*} \mathrm{u}+3^{*} \mathrm{c} 0^{*} \operatorname{sqrt}(\mathrm{v})\right)$ :

\#4. Compute c0 (c0A) the adjusted critical value

$\mathrm{k} 1:=0: \mathrm{k} 3:=5.9$ :

for $\mathrm{i}$ from 1 to 100

do $\mathrm{k} 2:=(\mathrm{k} 1+\mathrm{k} 3) / 2: \mathrm{c} 0:=\operatorname{evalf}(\mathrm{k} 2)$ :

\#4.1. Find K0, lower integration bound

$\mathrm{K} 0:=0: \mathrm{z} 1:=\operatorname{evalf}(\mathrm{H}(\mathrm{c} 0, \mathrm{~K} 0))$ :

if $\mathrm{z} 1<1 \mathrm{e}-8$ then for $\mathrm{j}$ from 1 to $\operatorname{trunc}(\mathrm{K})$

do $\mathrm{z} 1:=\operatorname{evalf}(\mathrm{H}(\mathrm{c} 0, \mathrm{j}))$;

if $\mathrm{z} 1>1 \mathrm{e}-8$ then for $\mathrm{h}$ from $\mathrm{j}-0.9$ by 0.1 to $\mathrm{j}$

do $\mathrm{z} 1:=\operatorname{evalf}(\mathrm{H}(\mathrm{c} 0, \mathrm{~h}))$;

if $\mathrm{z} 1>1 \mathrm{e}-8$ then $\mathrm{K} 0:=\mathrm{h}-0.1$ :break fi:

od:

break fi:

od:

fi:

\#4.2. Find K1, upper integration bound $\mathrm{K} 1:=\operatorname{evalf}(\mathrm{K})-0.00001: \mathrm{z} 2:=\operatorname{evalf}(\mathrm{H}(\mathrm{c} 0, \mathrm{~K} 1))$ : if $\mathrm{z} 2<1 \mathrm{e}-8$ then for $\mathrm{j}$ from $\mathrm{K} 1$ by -1 to 0 do $\mathrm{z} 2:=\operatorname{evalf}(\mathrm{H}(\mathrm{c} 0, \mathrm{j}))$;

if $z 2>1 \mathrm{e}-8$ then for $\mathrm{h}$ from $\mathrm{j}+0.9$ by -0.1 to $\mathrm{j}$

do $\mathrm{z} 2:=\operatorname{evalf}(\mathrm{H}(\mathrm{c} 0, \mathrm{~h}))$;

if $\mathrm{z} 2>1 \mathrm{e}-8$ then $\mathrm{K} 1:=\mathrm{h}+0.1$ :break fi:

od:

break fi:

od:

fi:

if $\mathrm{z} 1<1 \mathrm{e}-8$ and $\mathrm{z} 2<1 \mathrm{e}-8$ then $\mathrm{y}:=0$

else $\mathrm{y}:=\operatorname{evalf}(\operatorname{int}(\mathrm{H}(\mathrm{c} 0, \mathrm{t})$,

$\mathrm{t}=\operatorname{evalf}(\mathrm{K} 0) .$. evalf $((\mathrm{K} 0+\mathrm{K} 1) / 2)))$

$+\operatorname{evalf}(\operatorname{int}(\mathrm{H}(\mathrm{c} 0, \mathrm{t}), \mathrm{t}=\operatorname{evalf}((\mathrm{K} 0+\mathrm{K} 1) / 2) . . \operatorname{evalf}(\mathrm{K} 1)))$ :

fi:

if $\mathrm{y}<$ alpha then $\mathrm{k} 3:=\mathrm{k} 2$ else $\mathrm{k} 1:=\mathrm{k} 2$ fi:

if $\operatorname{abs}(y-a l p h a)<0.00001$ then break fi:

od:

\#5. Print the adjusted critical value.

printf("the adjusted critical value is $\% .3 \mathrm{f}$

with $\% .2$ f per cent risk",c0,100*alpha);

\#6. Plot the power test

$\mathrm{ks} 1:=\left(-\mathrm{Cp} * \mathrm{u}^{*}(1-\mathrm{abs}(\right.$ delta $)) / 3+\operatorname{sqrt}\left(\mathrm{Cp}^{\wedge} 2^{*}\left(\mathrm{u}^{*}(1-\right.\right.$

$\operatorname{abs}($ delta $)) / 3)^{\wedge} 2+\left(\mathrm{Cp}^{\wedge} 2-\mathrm{Cpuv}^{\wedge} 2\right)^{*}\left(\mathrm{v}^{*} \mathrm{Cpuv}^{\wedge} 2\right.$

$\left.\left.\left.-\left(\mathrm{u}^{*}(1-\operatorname{abs}(\text { delta })) / 3\right)^{\wedge} 2\right)\right)\right) /\left(\mathrm{v}^{*} \operatorname{Cpuv}^{\wedge} 2-\left(\mathrm{u}^{*}(1-\right.\right.$

$\operatorname{abs}($ delta $\left.)) / 3)^{\wedge} 2\right)$ :

$\mathrm{kGs} 1:=\mathrm{ks} 1 / \operatorname{sqrt}\left(1+\operatorname{lambda}{ }^{\wedge}{ }^{*} \operatorname{Cp}^{\wedge} 2 /(1-\operatorname{abs}(\text { delta }))^{\wedge} 2\right)$ :

$\mathrm{kG} 1:=$ piecewise $(\mathrm{kG}>0,(1$-delta $) * \mathrm{kGs} 1,-(1+$ delta $) * \mathrm{kGs} 1)$ : 
$\mathrm{f}:=\mathrm{t}->(1$-delta $) *$ stats[statevalf,pdf,normald] $((1-$ delta $) * \mathrm{t}-$ $\left.\mathrm{kG} 1 * \mathrm{~N}^{\wedge} .5\right)+(1+$ delta $) *$ stats[statevalf,pdf,normald] $\left((1+\right.$ delta $\left.) * \mathrm{t}+\mathrm{kG} 1 * \mathrm{~N}^{\wedge} .5\right)$ : $\mathrm{pw}:=\mathrm{c} 0->\operatorname{int}(\mathrm{F}(\mathrm{c} 0, \mathrm{t}) * \mathrm{f}(\mathrm{t}), \mathrm{t}=0 . . \mathrm{K})$ :

$\operatorname{plot}(\mathrm{pw}(\mathrm{c} 0), \mathrm{Cpuv}=\mathrm{c} . \mathrm{Cp}-0.01, \mathrm{title}=$ "test power" $)$;

\section{References}

1. S. Kotz, N.L. Johnson, Process capability indices (Chapman and Hall, London, 1993)

2. D.R. Bothe, Measuring process capability (MC Graw Hill, New York, 1997)

3. S. Kotz, C.R. Lovelace, Process capability indices in theory and practice (Arnold, London, 1998)

4. W.L. Pearn, S. Kotz, Encyclopedia and handbook of process capability indices (World Scientific Publishing, Singapore, 2006), Vol. 12

5. V.E. Kane, Process capability indices, J. Qual. Tech. 18, 41-52 (1986)

6. L.K. Chan, S.W. Cheng, F.A. Spiring, A new measure of process capability: $\mathrm{C}_{p m}$, J. Qual. Tech. 20, 162-175 (1988)

7. W.L. Pearn, S. Kotz, N.L. Johnson, Distributional and inferential properties of process capability indices, J. Qual. Tech. 24, 216-231 (1992)

8. K. Vännman, A unified approach to capability indices, Stat. Sinica 5, 805-820 (1995)

9. R.A. Boyles, Process capability with asymmetric tolerances, Comm. Stat. Sim. Comp. 23, 615-643 (1994)

10. K.S. Chen, W.L. Pearn, Capability indices for processes with asymmetric tolerances, J. Chin. Inst. Eng. 24, 559 568 (2001)

11. D. Grau, Process yield and capability indices, Commun. Stat. Theory. Methods 40, 2751-2771 (2011)

12. D.C. Montgomery, G.C. Runger, Gauge capability and designed experiments. Part I: basic methods, Qual. Eng. 6, 115-135 (1993)

13. D.C. Montgomery, G.C. Runger, Gauge capability and designed experiments. Part II: experimental design models and variance component estimation, Qual. Eng. 6, 289305 (1993)
14. W.A. Levinson, How good is gauge? Semicond. Int. 165168 (1995)

15. H.J. Mittag, Measurement error effects on the performance of process capability indices, Front. Stat. Qual. Control 5, 195-206 (1997)

16. S. Bordignon, M. Scagliarini, Statistical analysis of process capability indices with measurement errors, Qual. Rel. Eng. Int. 18, 321-332 (2002)

17. S. Bordignon, M. Scagliarini, Statistical analysis of process capability indices with measurement errors: the case of $\mathrm{Cp}$, Stat. Meth. Appl. 10, 273-285 (2001)

18. S. Bordignon, M. Scagliarini, Estimation of Cpm when measurement errors is present, Qual. Rel. Eng. Int. 22, 787-801 (2006)

19. W.L. Pearn, M.Y. Liao, Measuring process capability based on $\mathrm{C}_{p k}$ with gauge measurement errors, Microelec. Rel. 45, 739-751 (2005)

20. W.L. Pearn, M.Y. Liao, One-sided process capability assessment in the presence of measurement errors, Qual. Rel. Eng. Int. 22, 771-785 (2006)

21. W.L. Pearn, M.Y. Liao, Estimating and testing process precision with presence of gauge measurement errors, Qual. Quant. 41, 757-777 (2007)

22. W.L. Pearn, M.H. Shu, B.M. Hsu, Testing process capability based on $\mathrm{C}_{p m}$ in the presence of random measurement errors, J. Appl. Stat. 32, 1003-1024 (2005)

23. B.M. Hsu, M.H. Shu, W.L. Pearn, Measuring process capability based on $\mathrm{C}_{p m k}$ with gauge measurement errors, Qual. Rel. Eng. Int. 23, 597-614 (2007)

24. D. Grau, Moments of the unbalanced non-central chisquare distribution, Stat. Prob. Lett. 79, 361-367 (2009)

25. W.L. Pearn, P.C. Lin, K.S. Chen, Estimating process capability index $C_{p m k}^{\prime \prime}$ for asymmetric tolerances: Distributional properties, Metrika 54, 261-279 (2001)

26. W.L. Pearn, P.C. Lin, Testing process performance based on capability index $\mathrm{C}_{p k}$ with critical values, Comp. Ind. Eng. 47, 351-369 (2004)

27. P.C. Lin, W.L. Pearn, Testing process performance based on the capability index $\mathrm{C}_{p m}$, Int. J. Adv. Manuf. Tech. 27, 351-358 (2005) 